\title{
Ozonización catalítica en el tratamiento de aguas residuales: Un análisis bibliométrico
}

INGENIERÍA QUÍMICA

\section{Catalytic ozonation in treatment of wastewater: $A$ bibliometric analysis}

\author{
Astrid C. Angel-Ospina ${ }^{1 \S}$ (D), Fiderman Machuca-Martínez ${ }^{1(D)}$ \\ ${ }^{1}$ Universidad del Valle, Escuela de Ingeniería Química, Cali, Colombia \\ §astrid.angel@correounivalle.edu.co, fiderman.machuca@correounivalle.edu.co
}

Recibido: 30 de septiembre de 2021 - Aceptado: 1 de diciembre de 2021

\begin{abstract}
Catalytic ozonation (OC) is a promising technology in terms of efficiency and energy cost as a treatment of industrial effluents that have pollutants of emerging concern, since it improves the oxidative capacities of ozone through catalysts that promote its decomposition and the generation of radicals oxidants species. This article studied the status and evolution of $\mathrm{OC}$ through a literature review and bibliometric analysis combined with the $\mathrm{S}$ curve technique to quantitatively analyze 1714 publications in SCOPUS and Scielo Index from 1973 to 2021.

The results showed that (1) the number of publications worldwide started in 1973 to have 189 documents in 2021; (2) China is the country with the highest number of publications and annual citations (958 and 19088, respectively) and it is a central country in international cooperation networks; (3) the analysis of keyword co-occurrence showed that research has prioritized OC reaction mechanisms, kinetic expressions, the catalysts, and since 2016, the pilot scale application with other advanced oxidation technologies; (4) The compound with larger research is oxalic acid and since 1999 ibuprofen and atrazine; (5) in Latin America, Brazil is the country with the most publications (32) and with the largest number of international cooperation networks and (5) Applied Catalysis B: Environmental and The Journal of the Brazilean Chemical Society (Latin America) are the journals with the best quartile that publish on OC. Finally, the $\mathrm{S}$ curve indicated that $\mathrm{OC}$ is in a growth stage in terms of the scientific maturity, exhibiting great potential in R\&D.
\end{abstract}

Keywords: bibliometric analysis, catalytic ozonation, S-curve, wastewater treatment, Emerging Contaminants. 


\section{Resumen}

La ozonización catalítica (OC) es una tecnología prometedora en términos de eficiencia y costo energético como tratamiento de efluentes industriales que poseen contaminantes de preocupación emergentes, ya que mejora las capacidades oxidativas del ozono a través de catalizadores que promueven su descomposición y la generación de especies radicales oxidantes, los cuales reaccionan con prácticamente cualquier compuesto presente en los efluentes. Este artículo estudió el estado y evolución de la OC mediante una revisión de la literatura y un análisis bibliométrico combinado con la técnica de curva S para analizar cuantitativamente 1714 publicaciones en SCOPUS y SciELO Index desde el año 1973 al 2021.

Los resultados mostraron que (1) el número de publicaciones a nivel mundial se inició en 1973 a tuvo 189 documentos en 2021; (2) China es el país con mayor número de publicaciones y citaciones anuales (958 y 19088, respectivamente) y es un país central en redes de cooperación internacional; (3) tanto el estado del arte como el análisis de co-ocurrencia de palabras clave mostró que la investigación ha priorizado mecanismos de reacción de la OC, expresiones cinéticas, catalizadores, y desde 2016, la aplicación a escala piloto en conjunto con otras tecnologías avanzadas de oxidación; (4) El compuesto con más investigaciones es el ácido oxálico y desde 1999 ibuprofeno y atrazina; (5) en América Latina, Brasil es el país con más publicaciones (32) y con mayor número de redes de cooperación internacional y (5) Applied Catalysis B: Environmental y Journal of the Brazilean Chemical Society (América Latina) son las revistas con mejor cuartil que publican sobre OC. Finalmente, la curva S indicó que la OC se encuentra en etapa de crecimiento en términos de madurez cientifica, exhibiendo un gran potencial en I+D.

Palabras clave: análisis bibliométrico, curva S, ozonización catalítica, tratamiento de aguas residuales, Contaminantes de Preocupación Emergente.

\section{Introducción}

En un contexto de contaminación y problemas de gestión del agua a nivel mundial, el acceso a un agua libre de contaminación de cualquier tipo ya sea para consumo humano o para actividades industriales y domésticas, se ha convertido en uno de los objetivos centrales de la humanidad en el siglo XXI, tanto es así, que forma parte de la agenda 2030 de objetivos de desarrollo sostenible (ODS) de la ONU ( ${ }^{\circ}$ 6: Agua limpia y saneamiento) ${ }^{(1)}$. En este sentido, desde el año 1960, se ha observado que el PIB mundial aumentó en promedio $3.5 \%$ anual hasta el año $2012^{(1,2)}$, Sin embargo, este mismo hecho ha provocado una demanda sin precedentes del agua para actividades industriales y se prevé que para el 2030, el mundo tendrá que enfrentarse a un déficit mundial del $40 \%$ de agua, acompañado además de un escenario de cambio climático del que ya se están observando sus efectos en distintas partes del mundo ${ }^{(3)}$. La Figura 1 muestra cómo está evolucionando la situación mundial del agua y cómo se pronostica para los próximos años una escasez mundial de agua.
Teniendo en cuenta esta preocupante situación una parte importante de la investigación mundial actual se ha orientado en desarrollar procesos de regeneración de aguas residuales para producir agua que pueda ser reutilizada con posterioridad a los tratamientos convencionales con el fin de 1) Mejorar las condiciones del agua consumida por los humanos y el acceso a ella 2) Disminuir la contaminación de cuerpos de agua naturales y la modificación de ecosistemas y 3) Reutilizar el agua residual producto de las actividades humanas con el fin de reducir la huella hídrica global ${ }^{(5,6)}$. El grado de regeneración de estas aguas por medio de tecnologías avanzadas va a depender de la utilización posterior que se les vaya a dar, además, de las condiciones de escasez, demanda y normatividad del país o región en el que se aplica el tratamiento ${ }^{(7,8)}$. La Figura 2 muestra las consecuencias del tratamiento $\mathrm{y}$ reutilización de aguas residuales y se observa que el tratamiento permite, no solo regenerar el agua residual, sino que también es capaz de generar valor agregado en un entorno de economía circular. 


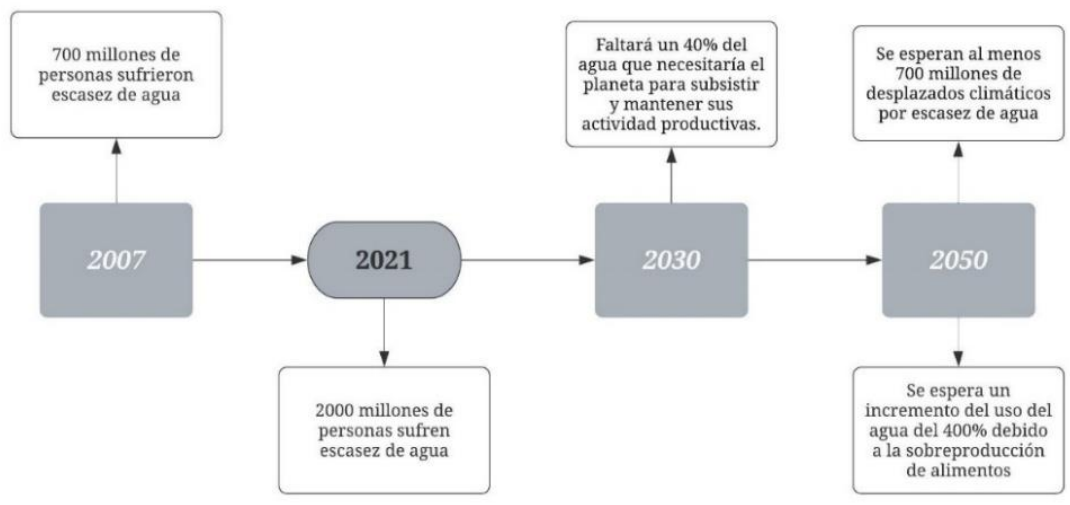

Figura 1. Situación actual del agua y perspectivas a nivel mundial. Fuente: propia con información adaptada de Tsiarta $N^{(4)}$

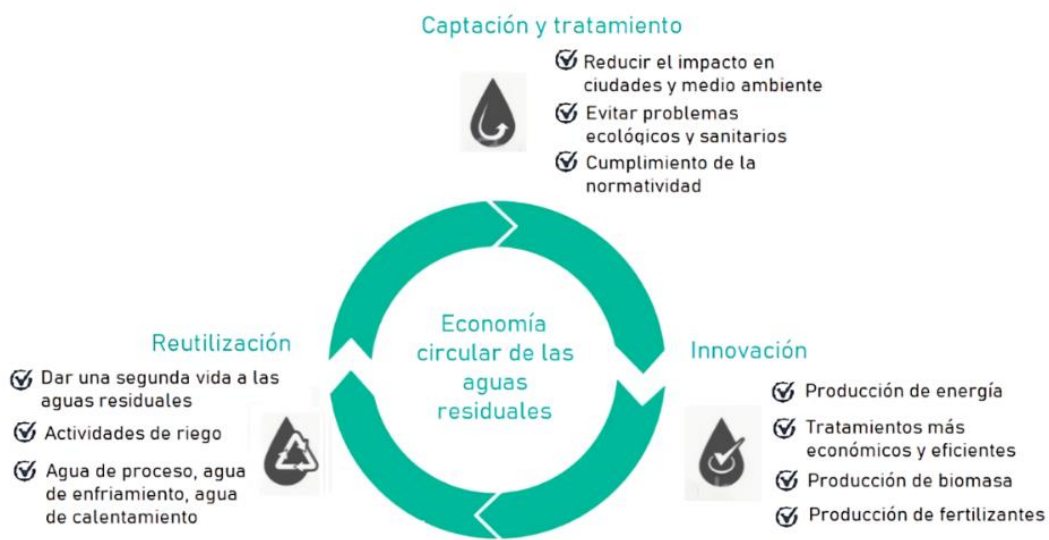

Figura 2. Soluciones para las aguas residuales. Fuente: propia

En consideración a lo anterior, el presente artículo se centra en abordar la contaminación de aguas residuales que están demasiado diluidas o poseen contaminantes difíciles de remover por procesos convencionales, lo que limita su reutilización. Estos contaminantes se denominan Contaminantes de Preocupación Emergentes o CECs por sus siglas en inglés (Químicos Persistentes, Bioacumulables y Tóxicos (PBTs), nanopartículas, pesticidas, residuos farmacéuticos y químicos, compuestos del cuidado personal (PCPs), etc.), los cuales representan una amenaza tanto para las fuentes hídricas, como para el consumo humano y demás actividades agrícolas e industriales ${ }^{(9,10)}$. Para los CECs han surgido en los últimos años tecnologías que permiten potenciar y/o mejorar la efectividad de los resultados alcanzados por tratamientos fisicoquímicos y biológicos convencionales, los cuales se enmarcan dentro del tratamiento terciario de aguas residuales.

Uno de los grupos especialmente atractivos de tecnologías que se incluyen dentro de estos tratamientos son los Procesos Avanzados de Oxidación (PAOs), que constituyen una familia de tecnologías similares, pero no idénticas, que se basan predominantemente (pero no exclusivamente) en la generación de radicales hidroxilo $(\bullet \mathrm{OH})$ por medios fotoquímicos (incluida la luz solar), u otras formas de energía. Estos radicales son altamente reactivos, sin mucha selectividad y con una alta efectividad para la oxidación de materia orgánica y algunos compuestos inorgánicos ${ }^{(11,12)}$. Entre estas 
tecnologías, la ozonización catalítica (OC) es una de las de mayor potencial, ya que aprovecha las cualidades oxidativas del ozono y las optimiza por medio de distintos catalizadores, permitiendo una mejora en la degradación de contaminantes. Debido a que la OC puede desarrollarse de manera tanto homogénea como heterogénea, la presente investigación investigará ambas modalidades, pero priorizando especialmente la OC heterogénea, que ha demostrado ser, entre las dos tecnologías, la más efectiva, limpia, económica y ambientalmente sostenible (13), convirtiéndose en uno de los PAO de más auge en la última década en el ámbito de la investigación en el uso de ozono ${ }^{(14,15)}$.

Para ello, el artículo investiga el estado y evolución de la OC a través de tres herramientas: una revisión de la literatura acompañado de un análisis de desempeño para identificar y sintetizar conceptos claves como cinéticas de reacción, catalizadores más comunes y nuevos desarrollos al año 2021; un análisis bibliométrico de la producción científica mundial a la fecha comparándola con el contexto latinoaméricano para determinar la situación de la $\mathrm{OC}$ en el panorama académico-científico, ya que no se ha observado un análisis similar; y, finalmente, con los resultados obtenidos se implementa una curva S para estimar el estado actual de la tecnología en términos de la evolución en investigación científica.

\section{Metodología}

Se realizó una exploración en las bases de datos Scopus y SciELO Index de Web of Science, con el fin de observar el estado de la investigación en OC. Se utilizaron las palabras clave (catal*) AND (ozon*) AND \{wastewater AND \{catalytic ozonation \} durante el período 1973-2021, excluyendo los resultados correspondientes a la fotocatálisis y limitando los resultados de OC a aguas residuales (ya que esta tecnología también se utiliza en aire).
El operador de truncamiento $\left(^{*}\right)$ permite incluir las variantes de los términos como "catalyst", "catalysts", "ozone", “ozonation", “ozonization", etc.; mientras que el operador \{\} permite refinar los resultados asegurando que en el artículo encontrado exista la frase exacta. Con los resultados obtenidos se realizó un análisis bibliométrico, una herramientas eficaz para el análisis cuantitativo y cualitativo de las actividades científicas, ya que la misma se centra en analizar la cantidad de características externas de la literatura (como artículos, libros y patentes) y referencias (citas y co-citas) mediante el uso de métodos estadísticos y matemáticos para descubrir patrones y tendencias, permitiendo manejar grandes volúmenes de datos científicos y producir un alto impacto en la investigación (16). El análisis bibliométrico se dividió en 3 partes:

\subsection{Análisis de desempeño y revisión de la literatura}

El análisis de desempeño examina las contribuciones de los componentes de la investigación al campo de la investigación ${ }^{(16)}$ y se abordó con métricas relacionadas con número total de citaciones de los artículos en orden de presentar una revisión de la literatura de la OC hasta el momento con los artículos más importantes. Para realizarlo se utilizó la herramienta "Smart Citations" de la Startup Scite, una inteligencia artificial que permite clasificar artículos en base al indicador SI (Índice Scite) ${ }^{(17)}$, el cual está definido por la Ec.1. El SI tiene en cuenta la confiabilidad de la publicación al comparar las citas de apoyo o respaldo recibidas por el artículo, frente a las citas por contradicción o debate. La Figura 3 muestra como funciona el indicador, teniendo en cuenta que el artículo fuente es el nodo más grande y de él salen todos los artículos citados (flechas negras). Las flechas (verdes) que llegan al nodo principal indican las investigaciones que apoyan o respaldan el artículo fuente, mientras que las flechas que salen del nodo (flecha azul) indican las menciones por 
contraste que debaten los resultados obtenidos por el estudio.

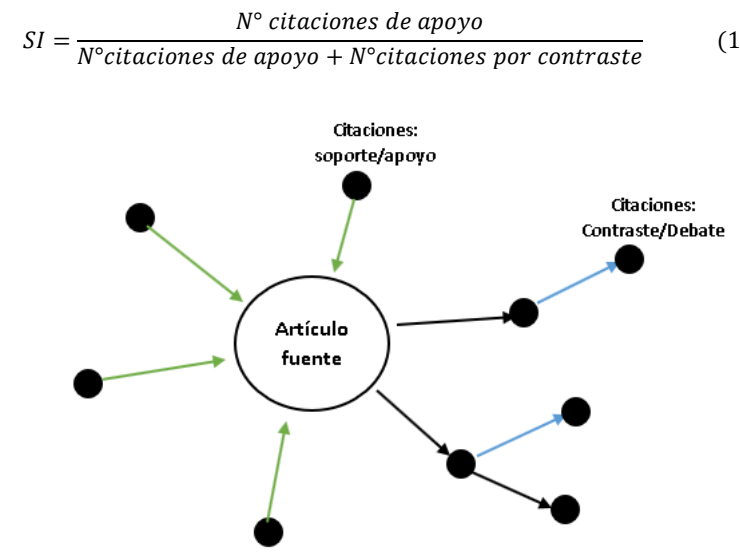

Figura 3. Funcionamiento del Índice SI de Scite. Fuente: propia con información adaptada de Scite (17).

\subsection{Mapeo científico $\mathrm{y}$ análisis de redes}

El mapeo científico es una parte del análisis bibliométrico que se centra en las relaciones entre los componentes de la investigación, como análisis de coautoría, análisis de cocitación, palabras clave, etc., mientras que el análisis de redes se realiza por medio de agrupamientos jerárquicos, escalamiento multidimensional, algoritmos de centrado, etc., para presentar los resultados en forma de clústeres ${ }^{(16)}$. Para realizar estos dos procedimientos se utilizó la herramienta VOSViewer v.1.6.16 ${ }^{(18)}$, la cual permitió generar redes bibliométricas a partir de los metadatos de los artículos seleccionados. Finalmente, la calidad de las revistas que reportaron mayor número de publicaciones y citaciones acerca de la OC se analizó mediante el índice SJR (Scimago Journal $\&$ Country Rank) ${ }^{(19)}$.

\subsection{Madurez tecnológica: Curva S}

Toda tecnología está supeditada a un proceso productivo desde la fase de introducción hasta la sustitución, una vez agotada sus capacidades. Esta caracterización de una tecnología atada a un proceso o a un conjunto de procesos es la base de los modelos de madurez tecnológica, los cuales intentan definir una evolución de las características de una tecnología a través de etapas secuenciales y bien definidas ${ }^{(20)}$. Dado que generalmente se espera que un producto $o$ tecnología siga una curva $\mathrm{S}$, muchos investigadores la han utilizado en los últimos años para clasificar la evolución tecnológica en términos de 4 fases: emergente, crecimiento, madurez y decaimiento (saturación) ${ }^{(20)}$. Para analizar cuantitativamente la madurez de la OC en términos de investigación científica se construyó la curva $\mathrm{S}$ con el software Loglet Lab 4 desarollado por la Universidad Rockefeller ${ }^{(21)}$ a través del modelo de Gompertz, el cual utiliza la Ec. 2:

$$
Y=\frac{K-d}{1+e^{-r(t-t m)}}+d
$$

donde Y representa la variable dependiente de la curva $\mathrm{S}$, es decir, las publicaciones acumuladas anualmente, $\mathrm{t}$ representa la variable de tiempo, $\mathrm{d}$ y $\mathrm{r}$ son parámetros del modelo, tm es el tiempo medio y $\mathrm{K}$ es el valor máximo de publicaciones.

\section{Resultados}

\subsection{Análisis de desempeño y revisión de la literatura}

\subsubsection{Ozonización}

El ozono $\left(\mathrm{O}_{3}\right)$ fue descubierto en 1840 , pero no fue hasta 1886 cuando se encontró que podía ser utilizado como germicida para la esterilización de agua contaminada ${ }^{(12)}$. En 1906 se instaló el primer sistema de desinfección de aguas residuales municipales con $\mathrm{O}_{3}$ en Niza (Francia) y desde entonces, ha sido ampliamente utilizado con fines de desinfección, sobre todo en Europa, ya que el cloro, normalmente utilizado en la desinfección (cloración), produce compuestos orgánicos halogenados que fueron identificados como agentes potenciales de riesgo para la salud pública ${ }^{(9)}$. De esta manera, el $\mathrm{O}_{3}$ funciona como un tratamiento complementario a la cloración, 
eliminando no solo patógenos, sino también materia orgánica para inhibir la producción de compuestos halogenados de desinfección, usualmente tóxicos, los cuales se producen durante la cloración ${ }^{(22)}$.

Debido a su alto poder oxidativo, el $\mathrm{O}_{3}$ se ha venido investigando como una tecnología de oxidación en el tratamiento de aguas residuales de uso industrial y doméstico para eliminar microorganismos, iones inorgánicos y contaminantes orgánicos; teniendo su mayor aplicación en la remoción de sabor y como coadyuvante en los procesos de coagulación y filtración ${ }^{(23)}$. Dependiendo de las condiciones del medio y del tipo de contaminante, el $\mathrm{O}_{3}$ actúa a través de dos tipos de reacción: directa o de tipo radical, los cuales se muestran en la Figura 4.

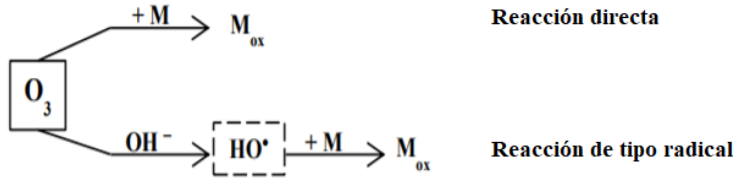

Figura 4. Vías de reacción del $\mathrm{O}_{3}$. Adaptado de Jeirani $Z^{(14)}$

La reacción directa se produce en puntos nucleofílicos por reacciones de adición en restos insaturados (enlaces de carbono dobles y triples), por transferencia de electrones o por transferencia de átomos de oxígeno, debido a que el $\mathrm{O}_{3}$ por sí solo tiene un alto potencial redox $(2.08 \mathrm{~V}){ }^{(22,24)}$. Los productos de estas reacciones son la materia orgánica oxidada, $\mathrm{O}_{2}$, diferentes iones como el hidroperóxido $\left(\mathrm{OH}_{2}^{-}\right)$y los ozonuros $\left(\mathrm{O}_{3}^{-}\right)$, radicales como el hidroperoxil $\left(\bullet \mathrm{OH}_{2}\right)$ y sustancias como el peróxido de hidrógeno $\left(\mathrm{H}_{2} \mathrm{O}_{2}\right)$ (24). La Ec.3 muestra la reacción global de una ozonización directa y valores aproximados para la constante de velocidad de reacción, con $\mathrm{S}$ siendo el contaminante, observándose que las reacciones directas de ozonización siempre son bimoleculares ${ }^{(25)}$ :

$$
O_{3}+S \rightarrow S_{O X} \quad ; \quad k \approx 1-100 M^{-1} S^{-1}
$$

Además de estas reacciones, en medio básico o a altas concentraciones de material orgánico disuelto, agregando $\mathrm{H}_{2} \mathrm{O}_{2}$ o por radiación UV, predomina el tipo de reacción indirecta o de tipo radical, en donde el $\mathrm{O}_{3}$ descompone para formar el radical $\bullet \mathrm{OH}$, el oxidante más fuerte disponible en el tratamiento del agua. Los radicales $\cdot \mathrm{OH}$ pueden oxidar material orgánico por transferencia de electrones, transferencia de hidrógenos o reacciones de adición ${ }^{(24)}$. La Ec.4 muestra la reacción global de una oxidación indirecta, y adicionalmente, valores aproximados para la constante de velocidad de reacción, en donde se observa que también es una reacción bimolecular (25):

$2 \mathrm{O}_{3}+\mathrm{H}_{2} \mathrm{O} \rightarrow 2 \mathrm{OH} \bullet+2 \mathrm{O}_{2}+\mathrm{OH}_{2}^{-} \bullet$

$k \approx 108-1010 M^{-1} S^{-1}$

A través de la Ec.4 la materia orgánica puede ser completamente mineralizada (reducida a su forma más simple, $\mathrm{CO}_{2}$ y $\mathrm{H}_{2} \mathrm{O}$ ), sin embargo, lo más común es que las reacciones posteriores conlleven la absorción de un átomo de $\mathrm{H}$ por el oxígeno disuelto y la formación de radicales hidroperoxilo, peróxido de hidrógeno y series de peroxilos y peróxidos, aldehídos, ácidos y demás subproductos (24) que suelen ser sustancias químicas más polares, y por lo tanto, más solubles en agua, menos volátiles, menos lipofílicas y menos absorbibles en filtros de carbón activado (CA). Aun así, estas sustancias (además de que tienden a ser menos olorosas y tóxicas) son, generalmente, más degradables por procesos microbiológicos, lo cual implica que la ozonización puede ir acompañada por un postratamiento, generalmente biológico, para eliminar estos subproductos (24). Otros subproductos de bajo peso molecular también pueden ser eliminados a través de CA y filtros de arena ${ }^{(24)}$.

La Tabla 1 muestra las constantes de velocidad del radical $\bullet \mathrm{OH}$ en comparación con el $\mathrm{O}_{3}$ de algunos compuestos orgánicos. Se observa que las 
constantes de velocidad de las reacciones que involucran radicales $\bullet \mathrm{OH}$ son mucho más altas, por lo cual, la reacción ocurre de manera mucho más rápida. Esta propiedad junto con la alta reactividad del radical $\bullet \mathrm{OH}$ con los contaminantes del medio son las que hacen atractivas las reacciones de ozonización indirectas.

Tabla 1. Constantes de velocidad en $\mathrm{Lmol}^{-1} \mathrm{~s}^{-1}$ del radical $\bullet \mathrm{OH}$ en comparación con el $\mathrm{O}_{3}$

\begin{tabular}{ccc}
\hline Compuesto & $\begin{array}{c}\text { Reacción } \\
\text { indirecta } \\
(\text { Radical } \cdot \mathbf{O H})\end{array}$ & $\begin{array}{c}\text { Reacción } \\
\text { directa }\left(\mathbf{O}_{3}\right)\end{array}$ \\
\hline Fenoles & $10^{9}-10^{10}$ & $10^{3}$ \\
Alquenos & $10^{9}-10^{11}$ & $10^{-1}-10^{3}$ \\
clorados & $10^{8}-10^{10}$ & $1-10^{2}$ \\
Aromáticos & $10^{9}-10^{10}$ & 1 \\
Cetonas & $10^{8}-10^{9}$ & $10^{-2}-1$ \\
Alcoholes & $10^{6}-10^{9}$ & $10^{-2}$ \\
Alcanos & Fuente: Sáenz $B^{(25)}$ \\
\hline
\end{tabular}

Debido al alto potencial oxidativo del radical $\bullet \mathrm{OH}$ $(2.80 \mathrm{~V})$, este es mucho más efectivo en la descomposición de compuestos presentes en el agua, sin embargo, no es selectivo y tiene una vida media muy corta. Adicionalmente, aunque la ozonización actúe en el medio a través de la reacción directa e indirecta, esta tecnología presenta dos problemas importantes: el $\mathrm{O}_{3}$ tiene baja solubilidad en agua y poca estabilidad química (se descompone rápidamente en $\mathrm{O}_{2}$ ) (26,27).

Para potenciar las ventajas del $\mathrm{O}_{3}$ y optimizar los procesos de tratamiento de aguas residuales, los PAOs combinan el $\mathrm{O}_{3}$ con otros compuestos químicos como el $\mathrm{H}_{2} \mathrm{O}_{2}$ (peróxono), y más recientemente, con catalizadores metálicos (ozonización catalítica) y con otro tipo de tecnologías como la luz ultravioleta (ozonización fotocatalítica) para mejorar las características del $\mathrm{O}_{3}$ e incrementar las tasas de oxidación de compuestos orgánicos y otros contaminantes inorgánicos ${ }^{(26)}$. La combinación del $\mathrm{O}_{3}$ con estas tecnologías permite favorecer y oxidar compuestos mediante la producción in situ de agentes radicales, especialmente del radical $\bullet \mathrm{OH}$ (4), el cual no es selectivo y, una vez es generado, reacciona de forma rápida y agresiva con la mayoría de las especies orgánicas presentes en el sistema. La Ec.5 muestra como sería la reacción de mineralización de un compuesto orgánico B por acción del radical $\bullet \mathrm{OH}^{(28)}$. Además, también se puede producir la oxidación de contaminantes inorgánicos hasta su estado de oxidación estable más alto ${ }^{(29)}$, los cuales pueden ser removidos luego por filtración ${ }^{(30)}$.

- $\mathrm{OH}+\mathrm{S} \rightarrow$ Intermedios $+\bullet \mathrm{OH} \rightarrow \mathrm{CO}_{2}+\mathrm{H}_{2} \mathrm{O}$

\subsubsection{Ozonización catalítica}

Wang et. al ${ }^{(12)}$ define la OC como el proceso en el cual se utilizan catalizadores que promueven la descomposición de $\mathrm{O}_{3}$ y generan radicales libres activos que mejoran la degradación y mineralización de contaminantes orgánicos sin la necesidad de utilizar luz o ajustar pH del medio. Esta tecnología se ha investigado de manera importante desde el siglo pasado. Hill et al. (31) llevó a cabo una investigación en 1945 en la que utilizó un catalizador de cobalto $\left(\mathrm{Co}^{2+}\right)$ y encontró que podía catalizar la descomposición de $\mathrm{O}_{3}$ para producir radicales $\bullet \mathrm{OH}$ en medio ácido (ácido acético o perclórico). Hewes y Davison ${ }^{(23,32)}$ por su parte, investigaron en 1972 el tratamiento de aguas residuales municipales utilizando metales de transición $\left(\mathrm{Co}^{2+}, \mathrm{Ti}^{2+}, \mathrm{Mn}^{2+}\right.$, etc) como catalizadores y encontraron buenos resultados en la remoción de carbono orgánico total (COT).

Actualmente, diversos estudios han encontrado que el uso de ozono junto con catalizadores de óxidos metálicos como $\mathrm{MnO}_{2}, \mathrm{TiO}_{2}, \mathrm{Al}_{2} \mathrm{O}_{3} \mathrm{u}$ óxidos metálicos soportados como $\mathrm{Cu}-\mathrm{Al}_{2} \mathrm{O}_{3}, \mathrm{Cu}$ $\mathrm{TiO}_{2}, \mathrm{Ru}-\mathrm{CeO}_{2}, \quad \mathrm{Fe}_{2} \mathrm{O}_{3} / \mathrm{Al}_{2} \mathrm{O}_{3}, \mathrm{~V}-\mathrm{O} / \mathrm{TiO}_{2}, \mathrm{~V}-$ $\mathrm{O} /$ silica gel y $\mathrm{TiO}_{2} / \mathrm{Al}_{2} \mathrm{O}_{3}{ }^{(26,33)}$, es un método efectivo para la remoción de contaminantes orgánicos en las aguas residuales ya que se presenta una aceleración significativa del proceso 
de degradación en presencia de estos catalizadores, sin embargo, a pesar de años de investigación, la tecnología no ha logrado aplicarse ampliamente en la industria. En este punto, es importante hacer la distinción sobre cómo se generan los radicales $\bullet \mathrm{OH}$ a partir de la OC y la forma en la que se puede presentar el catalizador en el medio, describiéndose las ventajas y desventajas de ambos métodos:

- Ozono Catalítico homogéneo: que utiliza $\mathrm{O}_{3}$ en fase acuosa junto a metales de transición disueltos como $\mathrm{Fe}$ (II), Mn (II), Ni (II), $\mathrm{Co}$ (II), Cd (II), Cu (II), $\mathrm{Ag}$ (I), Cr (III) y Zn (II). La elección del metal de transición aplicado determina no solo la velocidad de reacción, sino también la selectividad y el consumo de ozono ${ }^{(26)}$.

- Ozono Catalítico heterogéneo: en donde existe un sistema de 3 fases (gas-sólidolíquido). La fase sólida puede ser un catalizador metálico (óxidos de manganeso, hierro, aluminio, titanio, etc) inmovilizado en un soporte y la fase líquida es el agua residual en donde se burbujea la fase gaseosa, que incluye el $\mathrm{O}_{3}$. La actividad de los catalizadores se basa principalmente en la descomposición catalítica del $\mathrm{O}_{3}$ y la generación mejorada de radicales $\bullet \mathrm{OH}$. El mecanismo de reacción propuesto para describir este método sigue un proceso de adsorción, oxidación, formación de radicales, formación de complejos intermedios y/o mineralización de los compuestos ${ }^{(34)}$, sin embargo, uno de los principales problemas es que se desconoce a ciencia cierta el mecanismo de reacción por el cual se producen las reacciones de adsorción, descomposición de $\mathrm{O}_{3} \quad \mathrm{y}$ generación de radicales $\bullet \mathrm{OH}$ en la superficie del catalizador, lo que representa una barrera fundamental para el escalamiento de esta tecnología a nivel industrial ${ }^{(35)}$.

\section{Ozono Catalítico homogéneo}

En la OC homogénea las especies metálicas están disueltas en el medio acuoso junto con el $\mathrm{O}_{3}$. Este tipo de ozonización se caracteriza porque ocurre la descomposición del ozono por especies metálicas activas ${ }^{(36)}$ en la que la especie metálica (generalmente metales de transición) reacciona con $\mathrm{O}_{3}$ para producir radicales $\bullet \cdot \mathrm{H}$. Por ejemplo, Yildirim et al. ${ }^{(37)}$ indica que el sistema $\mathrm{O}_{3} / \mathrm{Fe}$ (II) involucra una reacción directa entre $\mathrm{Fe}$ (II) y $\mathrm{O}_{3}$ como se indica en las Ec. 6 y 7 :

$$
\begin{aligned}
& \mathrm{Fe}^{2+}+\mathrm{O}_{3} \rightarrow \mathrm{FeO}^{2+}+\mathrm{O}_{2} \\
& \mathrm{FeO}^{2+}+\mathrm{H}_{2} \mathrm{O}^{+} \rightarrow \mathrm{Fe}^{3+}+\cdot \mathrm{OH}+\mathrm{OH}^{-}
\end{aligned}
$$

Los autores compararon la mineralización del tinte azoico RR194 a través de ozonización y OC homogénea y se observó que esta última supera los resultados obtenidos por ozonización en cuanto a eficiencia y tiempo de mineralización. En este tipo de sistema la concentración de Fe(II) debe ser baja, ya que a altas concentraciones este reacciona con $\mathrm{FeO}^{2+}$, consumiendo el catalizador en el proceso y transformándolo en $\mathrm{Fe}(\mathrm{III})$ a través de una reacción secundaria no deseada ${ }^{(37)}$. Esto es una limitación importante que tiene lugar en la OC homogénea.

Otra ruta de acción a tener en cuenta es que los catalizadores no sólo reaccionan con el $\mathrm{O}_{3}$ para producir radicales $\bullet \mathrm{OH}$, sino que algunos también tienen la capacidad de adsorber materia orgánica y promover el contacto con $\mathrm{O}_{3}{ }^{(38,39)}$, sin embargo, aún con las ventajas que tiene el proceso, la OC homogénea generalmente reporta altos costes de operación, debido a que no solamente se tienen costes en la generación de $\mathrm{O}_{3}$, sino también en las unidades de recuperación del catalizador. En este sentido, Ziylan et al. ${ }^{(36)}$ analiza las dificultades en la recuperación del catalizador y su estudio es respaldado más recientemente por el artículo de Wang et al. ${ }^{(12)}$ al concluir que la OC homogénea no permite la recuperación efectiva del 
catalizador y, además, utiliza iones de metales pesados que pueden resultar tóxicos aún en muy bajas concentraciones, si permanecieran en el agua luego del proceso de recuperación del catalizador. Lo anterior ha llevado a que, en los últimos años, las investigaciones se hayan centrado cada vez más en la OC heterogénea.

A pesar de esto, la OC homogénea sigue siendo investigada y en los últimos años se han realizado varios estudios a escala de laboratorio sobre esta tecnología en muestras de aguas residuales contaminadas con colorantes, sales, ibuprofeno y otros compuestos farmaceúticos, aguas residuales municipales, efluentes del proceso de blanqueo de papel y efluentes de la industria lechera, con buenos resultados en la reducción de variables como la DQO (Demanda Química de Oxígeno), la DBO (Demanda Biológica de Oxígeno) y el COT. Es importante resaltar que todos estos estudios han sido realizados a escala de laboratorio solamente ${ }^{(40-44)}$. Un resumen de los catalizadores identificados se muestra en la Tabla 2.

\section{Ozono Catalítico heterogéneo}

En este tipo de ozonización el catalizador está presente en forma sólida, por lo que se tiene un sistema trifásico en donde ocurre la mineralización del contaminante por la ruptura de enlaces covalentes y la subsecuente formación de $\mathrm{CO}_{2}, \mathrm{H}_{2} \mathrm{O}$ y formas oxidadas de heteroátomos (como nitratos, fosfatos o sulfatos). En este sentido, según Crousier et al. ${ }^{(45)}$ y Yu et al. ${ }^{(46)}$, en la OC heterogénea se producen 5 fenómenos fisicoquímicos principales:

1) Transferencia de $\mathrm{O}_{3}$ de la fase gaseosa a la fase líquida.

2) Difusión de moléculas disueltas $\left(\mathrm{O}_{3}\right.$ y materia orgánica) hacia los sitios activos de la superficie del catalizador.
3) Adsorción de $\mathrm{O}_{3}$ disuelto y de especies orgánicas.

4) Reacciones químicas sobre la superficie del catalizador.

5) Desorción de los productos de reacción.

Aunque en el proceso de adsorción se produce una oxidación de la materia orgánica atrapada en los sitios activos del catalizador, la tasa de adsorción de esta etapa del proceso, en la mayoría de los artículos publicados, es bastante baja, por lo que el factor determinante de este proceso es en realidad la actividad del catalizador que provoca la descomposición de $\mathrm{O}_{3}$ en radicales $\bullet \mathrm{OH}^{(37)}$.

La OC heterogénea ha sido ampliamente estudiada en los últimos años porque permite la recuperación del catalizador de manera más eficiente $\mathrm{e}$ induce un menor riesgo de envenenamiento del catalizador para su posterior reutilización, de igual manera, es más efectiva en la generación de radicales $\cdot \mathrm{OH}$ y permite una mineralización más rápida de la materia orgánica presente en el agua residual ${ }^{(12)}$ en comparación con la OC homogénea. Finalmente, la OC heterogénea es ventajosa debido a las reacciones que se suceden de manera continua y sostenida a través de la superficie activa del catalizador, que no requiere un suministro continuo de reactivos (47).

Una de las áreas que más ha recibido investigación es la relacionada con el mecanismo de reacción de la OC heterogénea, ya que el modelamiento de las reacciones de adsorción que tienen lugar sobre la superficie del catalizador ha sido una tarea ardua y de discusión exhaustiva. Algunos estudios que se centraron en investigar la cinética de la OC heterogénea son los de Beltrán et. al ${ }^{(28,48)}$, los cuales muestran un acercamiento al mecanismo de reacción al tratar un agua contaminada con ácido oxálico a través de un catalizador combinado de $\mathrm{Co}_{3} \mathrm{O}_{4} / \mathrm{Al}_{2} \mathrm{O}_{3}$ y con un catalizador a base de CA, proponiendo que el sustrato $\mathrm{S}$ (ácido oxálico) atraviesa las 5 etapas 
antes mencionadas. Estos autores también establecen que en el medio no sólo ocurren reacciones de degradación de tipo heterogéneo sino también homogéneo $\mathrm{y}$, por lo tanto, la expresión de la velocidad de reacción de degradación del sustrato $S$ tiene la siguiente forma (Ec.8):

$$
-\frac{d C_{S}}{d t}=r_{T}=r_{H O M}+r_{H E T}
$$

Siendo $\mathrm{r}_{\mathrm{T}}$ la velocidad de degradación total que se expresa como la suma de las contribuciones de la velocidad de degradación por métodos homogéneos y heterogéneos. Al hablar de descomposición homogénea, estos autores se refieren a que el $\mathrm{O}_{3}$ se descompone en el medio acuoso a través de reacciones directas (Ec.3). Luego, la superficie del catalizador se hidroxila y los sitios activos del catalizador se convierten en puntos en donde se generan radicales $\bullet \mathrm{OH}$, como se indica en la Ec.9. Es importante tener en cuenta que la generación de radicales $\cdot \mathrm{OH}$ puede provenir, tanto de la superficie del catalizador (forma heterogénea) como de la descomposición del ozono en medio acuoso (forma homogénea), dependiendo de las condiciones de $\mathrm{pH}$, presencia de $\mathrm{H}_{2} \mathrm{O}_{2}$ o no, etc. Por ello, aun cuando el catalizador está en forma heterogénea, realmente se producen los dos tipos de OC.

$$
\mathrm{O}_{3}+\mathrm{OH}-\mathrm{S} \leftrightarrow \bullet \mathrm{O}_{3}-\mathrm{S}+\bullet \mathrm{OH}
$$

Otros estudios más recientes sobre el mecanismo de reacción de la OC heterogénea respaldan los estudios realizados por Beltrán et al. Huang et al. ${ }^{(49)}$ propone que la reacción de descomposición de ftalato de dibutilo (A) en solución acuosa en presencia de CA cargado con hierro sigue la expresión de la Ec.10, que se asemeja en gran medida a la propuesta por Beltrán et al. en la Ec. 8, donde el sustrato A se degrada por la acción combinada de $\mathrm{O}_{3}$ (ozonización directa o descomposición homogénea) y el radical $\cdot \mathrm{OH}$ (ozonización indirecta o descomposición heterogénea). En la $\quad$ Ec.10 $\quad \mathrm{kO}_{3} \quad \mathrm{y} \quad \mathrm{k} \cdot \mathrm{OH}$ representan las constantes de reacción de A con respecto a $\mathrm{O}_{3}$ y $\bullet \mathrm{OH}$, respectivamente.

$$
-\frac{d C_{A}}{d t}=k_{O_{3}}\left[O_{3}\right] C_{A}+k_{\bullet O H}[\bullet O H] C_{A}
$$

Seguidamente, otra área de estudios de OC heterogénea es la relacionada con la naturaleza y síntesis del catalizador, los cuales muestran tanto la efectividad de la OC heterogénea en diferentes tipos de aguas residuales (resultados resumidos en la Tabla 2), como el uso de catalizadores a base de materiales novedosos. Investigaciones como las de Nemati et al. ${ }^{(50)}$ y Li et al. ${ }^{(51)}$ estudian la efectividad de catalizadores hechos con nanopartículas de gama-alúmina $\left(\gamma-\mathrm{Al}_{2} \mathrm{O}_{3}\right)$ dopadas con cerio en un método de impregnacióncalcinación, encontrando eficiencias de remoción entre el $47 \%$ a $93 \%$; mientras que $\mathrm{Hu}$ et al. ${ }^{(52)}$ utiliza un catalizador de $\mathrm{Co}_{3} \mathrm{O}_{4}$ (nanopartículas de óxido de cobalto) combinado con aerogel macroporoso de carbono (MCA) para la reutilización de un agua real altamente contaminada de la industria textil, el cual le permitió 1) mejorar hasta en un $30 \%$ la remoción de DQO del efluente y 2) reutilizar el agua residual hasta 2 veces sin sacrificar la calidad de la tela.

Finalmente, Kolosov et al. ${ }^{(53)}$ realizó estudios de OC en la remoción de E.Coli y CECs (como atrazina, ibuprofeno, naproxeno y gemfibrozil) utilizando 2 tipos de catalizador: el Polonite $\AA$, un mineral utilizado comúnmente en la construcción de casas, llamado Opoka ${ }^{(54)}$ y la wollastonita, un mineral de inosilicato de calcio $\left(\mathrm{CaSiO}_{3}\right)$; luego, compara sus resultados con otros catalizadores convencionales basados en $\mathrm{AlO}_{2}, \mathrm{TiO}_{2}-\mathrm{Al}_{2} \mathrm{O}_{3}$ y zeolitas. Se observó que el Polonite®, la $\mathrm{AlO}_{2}$ y el $\mathrm{TiO}_{2} / \mathrm{Al}_{2} \mathrm{O}_{3}$ proporcionaron mecanismos para disminuir la dosis de $\mathrm{O}_{3}$ requeridas para alcanzar la desinfección, sin embargo, a diferencia de los dos últimos, el Polonite ${ }^{\circledR}$ no mostró un efecto perceptible en la remoción de CECs. Por otro lado, tanto la wollastonita como la zeolita, no mostraron resultados perceptibles en la remoción 
ni de E.Coli ni de CECs. A pesar de estos resultados, los autores concluyen que la combinación del Polonite ${ }^{\circledR}$ y el $\mathrm{TiO}_{2} / \mathrm{Al}_{2} \mathrm{O}_{3}$ entre ellos o con otros catalizadores diferentes, pueden incrementar su actividad catalítica, además de que son más susceptibles a la reutilización debido a su resistencia al envenenamiento y la degeneración.

Teniendo en cuenta las definiciones anteriores, se presenta la Tabla 2 que resume las ventajas y desventajas de la OC homogénea y heterogénea, los catalizadores más comunes utilizados en ambos tipos de OC y algunos estudios en donde se han aplicado. Seguidamente, se identificaron los 5 artículos más importantes de revisión (Tabla 2) y de investigación (Tabla 3); para cada cita se calculó el índice SI a través de la Ec.1 y se observó que la mayoría de ellos tuvieron citaciones de apoyo sin menciones por contradicción (100\%), lo que valida la confiabilidad de la información presentada. Otros artículos tuvieron un índice SI del 0\%, lo cual indica que no han recibido hasta el momento menciones de apoyo o contraste.

\subsubsection{Contexto latinoamericano}

La situación respecto a las aguas residuales en América Latina es un factor a tener en cuenta, ya que, en promedio, cerca del $70 \%$ de las aguas residuales vuelven a los ríos sin ser tratadas ${ }^{(76)}$. Otras estadísticas del banco mundial exponen que sólo el $60 \%$ de la población latinoamericana está conectada a un sistema de alcantarillado y un 30 $40 \%$ del agua residual que se capta, se trata realmente ${ }^{(14)}$. Al comparar estos indicadores con el promedio de países de la OCDE, se observa que el $81 \%$ de la población de estos países está conectada a un sistema de alcantarillado y el $77 \%$ de las personas se benefician del tratamiento de las aguas residuales al estar conectadas una planta específica (76), lo que evidencia una situación preocupante a en el contexto latinoamericano. Estos datos permiten asegurar que las diferencias en el desarrollo de políticas aplicadas a la mejora en la utilización del recurso hídrico de los países latinoamericanos, en comparación con países desarrollados, provocarán en el futuro implicaciones importantes en la salud pública, la sostenibilidad ambiental y la equidad social.

En este sentido, la ONU publicó en el año 2017 el reporte "Reutilizacion de aguas para agricultura en América Latina y el Caribe: estado, principios y necesidades" (77), en donde se expone que el país con mayor producción y tratamiento de aguas residuales es Brasil, seguido por México y luego Colombia. Del mismo informe se extrajo la Figura 5 que muestra cómo se encuentra la reutilización de aguas residuales en la región.

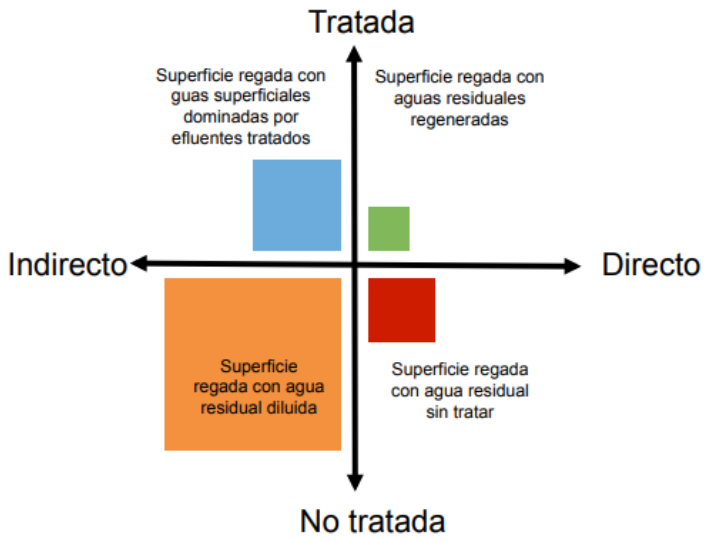

Figura 5. Reutilización de aguas residuales municipales en América Latina. Fuente: adaptado de Mateo-Sagasta ${ }^{\text {(77) }}$

Se observa que las aguas residuales pueden ser tratadas o no y se pueden reutilizar de manera directa (con poca o ninguna dilución de agua limpia) o indirectamente, una vez que se han vertido a otros cuerpos hídricos. Los cuadros naranja, azul, verde y rojo indican en proporción qué el agua residual más reutilizada en la región se hace de forma indirecta (el agua residual se vierte en ríos, por ejemplo) y sin ningún tipo de tratamiento, a excepción de la dilución con agua limpia. Esta es una situación preocupante debido a que ya se ha demostrado la presencia de CECs en agua potable $y$ residual en altas concentraciones en ciudades principales de la región como México, Bogotá, Rio de Janeiro, Minas Gerais y Santiago de Chile ${ }^{(78)}$. 
Tabla 2. Ventajas y desventajas de la OC y principales catalizadores

\begin{tabular}{|c|c|c|c|c|c|}
\hline Tipo de OC & Ventajas & Desventajas & $\begin{array}{c}\text { Tipo de } \\
\text { catalizador }\end{array}$ & $\begin{array}{c}\text { Composición del } \\
\text { catalizador }\end{array}$ & Referencia \\
\hline $\begin{array}{c}\text { OC } \\
\text { homogénea }\end{array}$ & $\begin{array}{l}\text { La presencia de iones } \\
\text { metálicos incrementa la } \\
\text { velocidad de reacción } \\
\text { entre el } \mathrm{O}_{3} \text { y el } \\
\text { contaminante }\end{array}$ & $\begin{array}{l}\text { Es necesario instalar } \\
\text { otra unidad para } \\
\text { recuperar el } \\
\text { catalizador }\end{array}$ & $\begin{array}{l}\text { Iones } \\
\text { metálicos } \\
\text { disueltos }\end{array}$ & $\begin{array}{l}\mathrm{Fe}(\mathrm{II}), \mathrm{Fe}(\mathrm{III}), \mathrm{Co}(\mathrm{II}), \\
\mathrm{Al}(\mathrm{III}), \mathrm{Mn}(\mathrm{II}), \mathrm{Cr}(\mathrm{III}), \\
\mathrm{Ag}(\mathrm{I}), \mathrm{Cu}(\mathrm{II}), \mathrm{Zn}(\mathrm{II}), \\
\mathrm{Cd}(\mathrm{II}), \mathrm{Pb}(\mathrm{II}), \mathrm{Ti}(\mathrm{II})\end{array}$ & $(36-44)$ \\
\hline \multirow{3}{*}{$\begin{array}{c}\text { OC } \\
\text { heterogénea }\end{array}$} & \multirow[b]{2}{*}{$\begin{array}{l}\text { Reutilización y } \\
\text { recuperación efectiva del } \\
\text { catalizador }\end{array}$} & $\begin{array}{l}\text { Literatura } \\
\text { contradictoria sobre } \\
\text { si los óxidos } \\
\text { metálicos catalizan o } \\
\text { no la formación de } \\
\text { radicales } \bullet \mathrm{OH}\end{array}$ & $\begin{array}{l}\text { Óxidos } \\
\text { metálicos }\end{array}$ & $\begin{array}{l}\mathrm{MnO} 2, \mathrm{~A} 12 \mathrm{O} 3, \mathrm{TiO} 2 \\
\mathrm{ZnO}, \mathrm{CeO} 2, \mathrm{Co} 3 \mathrm{O} 4, \mathrm{CuO}, \\
\mathrm{NiO}, \mathrm{SnO} 2, \mathrm{MgO}, \mathrm{Fe} 2 \mathrm{O} 3, \\
\mathrm{FeOOH}\end{array}$ & $(55-58)$ \\
\hline & & $\begin{array}{l}\text { No se conoce con } \\
\text { exactitud el } \\
\text { mecanismo de } \\
\text { reacción }\end{array}$ & $\begin{array}{c}\text { Materiales no } \\
\text { metálicos y } \\
\text { minerales }\end{array}$ & $\begin{array}{l}\text { CA (Carbon activado), } \\
\text { CNT (Nanopartículas de } \\
\text { carbono), MWCNT } \\
\text { (Nanotubos de carbono), } \\
\text { GO (Óxido de grafeno), } \\
\text { MCA (Aerogel } \\
\text { macroporoso de carbono), } \\
\text { Zeolita, Perovskita, } \\
\text { goetita, y otros minerales }\end{array}$ & $(52,53,59-65)$ \\
\hline & $\begin{array}{l}\text { Mayor cantidad de } \\
\text { materiales utilizados } \\
\text { como catalizadores }\end{array}$ & $\begin{array}{l}\text { Existen múltiples } \\
\text { experimentos a } \\
\text { diferentes } \mathrm{pH}, \text { lo que } \\
\text { dificulta la } \\
\text { uniformidad de los } \\
\text { resultados respecto al } \\
\text { mecanismo de } \mathrm{Rx}\end{array}$ & $\begin{array}{l}\text { Metáles / } \\
\text { Óxidos } \\
\text { metálicos } \\
\text { soportados }\end{array}$ & $\begin{array}{l}\mathrm{MnCeOx} / \gamma-\mathrm{Al} 2 \mathrm{O} 3, \beta- \\
\mathrm{FeOOH} / \mathrm{Al} 2 \mathrm{O} 3, \mathrm{CeO} 2 / \\
\text { OCNT } \\
\mathrm{Cu}, \mathrm{Ru}, \mathrm{Pt}, \mathrm{Co} \text { soportados } \\
\text { en } \mathrm{SiO} 2, \mathrm{Al} 2 \mathrm{O} 3, \mathrm{TiO} 2, \\
\mathrm{CeO} 2, \mathrm{CA}\end{array}$ & $(50,51,66,67)$ \\
\hline
\end{tabular}

Fuente: propia con información de Scopus

Tabla 3. Top 5 artículos de revisión sobre OC con índice SI

\begin{tabular}{|c|c|c|c|c|c|c|}
\hline Nombre del artículo & Autores & Fecha & $\begin{array}{c}\mathbf{N}^{\circ} \text { de } \\
\text { citaciones }\end{array}$ & $\begin{array}{c}\text { Índice SI } \\
(\%)\end{array}$ & Revista & Referencia \\
\hline $\begin{array}{l}\text { Catalytic ozonation and methods } \\
\text { of enhancing molecular ozone } \\
\text { reactions in water treatment }\end{array}$ & $\begin{array}{l}\text { Kasprzyk- } \\
\text { Hordern B, Ziółek } \\
\text { M, Nawrocki J }\end{array}$ & 2003 & 1127 & 100 & $\begin{array}{l}\text { Applied Catalysis B: } \\
\text { Environmental }\end{array}$ & (26) \\
\hline $\begin{array}{l}\text { The efficiency and mechanisms } \\
\text { of catalytic ozonation }\end{array}$ & $\begin{array}{l}\text { Nawrocki J, } \\
\text { Kasprzyk- } \\
\text { Hordern B }\end{array}$ & 2010 & 664 & 100 & $\begin{array}{l}\text { Applied Catalysis B: } \\
\text { Environmental }\end{array}$ & (38) \\
\hline $\begin{array}{l}\text { Heterogeneous catalytic } \\
\text { degradation of phenolic } \\
\text { substrates: Catalysts activity }\end{array}$ & $\begin{array}{l}\text { Liotta LF, } \\
\text { Gruttadauria M, } \\
\text { Di Carlo G, } \\
\text { Perrini G, } \\
\text { Librando V }\end{array}$ & 2009 & 339 & 100 & $\begin{array}{l}\text { Journal of Hazardous } \\
\text { Materials }\end{array}$ & (71) \\
\hline $\begin{array}{l}\text { Fe-based catalysts for } \\
\text { heterogeneous catalytic ozonation } \\
\text { of emerging contaminants in } \\
\text { water and wastewater }\end{array}$ & Wang J, Bai Z & 2017 & 315 & 100 & $\begin{array}{l}\text { Chemical } \\
\text { Engineering Journal }\end{array}$ & (72) \\
\hline $\begin{array}{l}\text { Catalytic ozonation for water and } \\
\text { wastewater treatment: Recent } \\
\text { advances and perspective }\end{array}$ & Wang J, Chen H & 2020 & 183 & 0 & $\begin{array}{l}\text { Science of the Total } \\
\text { Environment }\end{array}$ & (13) \\
\hline
\end{tabular}

Fuente: propia con información de Scopus 
Tabla 4. Top 5 artículos de investigación científica y tecnológica sobre OC con índice SI

\begin{tabular}{|c|c|c|c|c|c|c|}
\hline Nombre del artículo & Autores & Fecha & $\begin{array}{c}\mathbf{N}^{\circ} \text { de } \\
\text { citaciones }\end{array}$ & $\begin{array}{c}\text { Índice SI } \\
(\%)\end{array}$ & Revista & Referencia \\
\hline $\begin{array}{l}\text { Catalytic ozonation: A } \\
\text { promising advanced oxidation } \\
\text { technology for water treatment }\end{array}$ & $\begin{array}{l}\text { Legube B, Karpel } \\
\text { Vel Leitner N }\end{array}$ & 1999 & 560 & 100 & Catalysis Today & (73) \\
\hline $\begin{array}{l}\text { Surface hydroxyl groups of } \\
\text { synthetic } \alpha \text {-FeOOH in } \\
\text { promoting radical dotOH } \\
\text { generation from aqueous } \\
\text { ozone: Property and activity } \\
\text { relationship }\end{array}$ & Zhang $\mathrm{T}$ et al & 2008 & 250 & 0 & $\begin{array}{l}\text { Applied Catalysis B: } \\
\text { Environmental }\end{array}$ & (55) \\
\hline $\begin{array}{l}\text { Activated carbon catalytic } \\
\text { ozonation of oxamic and oxalic } \\
\text { acids }\end{array}$ & $\begin{array}{l}\text { Faria PCC, Órfão } \\
\text { JJM, Pereira MFR }\end{array}$ & 2008 & 249 & 100 & $\begin{array}{l}\text { Applied Catalysis B: } \\
\text { Environmental }\end{array}$ & (74) \\
\hline $\begin{array}{l}\text { Catalytic oxidation of benzene } \\
\text { with ozone over alumina- } \\
\text { supported manganese oxides }\end{array}$ & $\begin{array}{l}\text { Einaga } \mathrm{H}, \\
\text { Futamura S }\end{array}$ & 2004 & 225 & 100 & Journal of Catalysis & (75) \\
\hline $\begin{array}{l}\text { Catalytic ozonation of oxalic } \\
\text { acid in an aqueous TiO2 slurry } \\
\text { reactor }\end{array}$ & $\begin{array}{l}\text { Beltrán FJ, Rivas } \\
\text { FJ, Montero-de- } \\
\text { Espinosa R }\end{array}$ & 2002 & 209 & 100 & $\begin{array}{l}\text { Applied Catalysis B: } \\
\text { Environmental }\end{array}$ & (57) \\
\hline
\end{tabular}

Fuente: propia con información de Scopus

Para hacer frente a este problema, se han identificado en los últimos años estudios relacionados con PAOs, entre ellas, la OC. Por ejemplo, en Colombia Castro et al. ${ }^{(79)}$ realizaron un estudio sobre la evaluación de la OC heterogénea en la degradación de diclofenaco en soluciones preparadas, obteniendo una disminución en el COD y la DQO de entre el 94\% y $89 \%$, respectivamente.

Observando la resolución No. 631 de 2015 se observa que en Colombia no existen regulaciones en base a las concentraciones máximas permitidas de CECs en aguas residuales ${ }^{(80)}$, sin embargo, estos compuestos pueden ser candidatos a regulación futura, dependiendo de las investigaciones realizadas sobre sus efectos potenciales en la salud y los datos de monitoreo con respecto a su incidencia ${ }^{(81)}$. De igual manera, en Brasil también se identificaron estudios sobre OC heterogénea en donde se utilizó para tratar efluentes ricos en melanoidina (compuesto que se genera al someter determinados alimentos a altas temperaturas) y lixiviados de rellenos sanitarios municipales ${ }^{(82,83)}$, con resultados de remoción de color y COT del $98 \%$ y $80 \%$, respectivamente.

\subsection{Mapeo científico y análisis de redes}

La Figura 6a y 6 b resume la evolución del total de publicaciones en la ventana de estudio estudiada y clasificada por país a nivel internacional y latinoamericano, respectivamente. La búsqueda reportó un total de 1714 artículos (SCOPUS y SciELO Index de Web of Science), de los cuales el $90 \%$ son de investigación científica y tecnológica, $5.8 \%$ son artículos de conferencia, $2.4 \%$ son de revisión, $0.64 \%$ son capítulos de libro y el restante otros documentos (artículos de revisión en conferencias, artículos de datos, cartas al editor, etc), con un máximo de 189 artículos publicados durante el año 2021 y con China como el país con más publicaciones a la fecha (967), seguido de Irán (124) y España (79). De estos artículos, en América Latina se reportó un total de 80 publicaciones desde el año 2002, con Brasil como el país con más publicaciones totales (32), seguido de México (18) y Chile (14). Finalmente, solo 13 de estos 80 artículos fueron publicados en revistas latinoamericanas, siendo el $100 \%$ de investigación científica y tecnológica. 


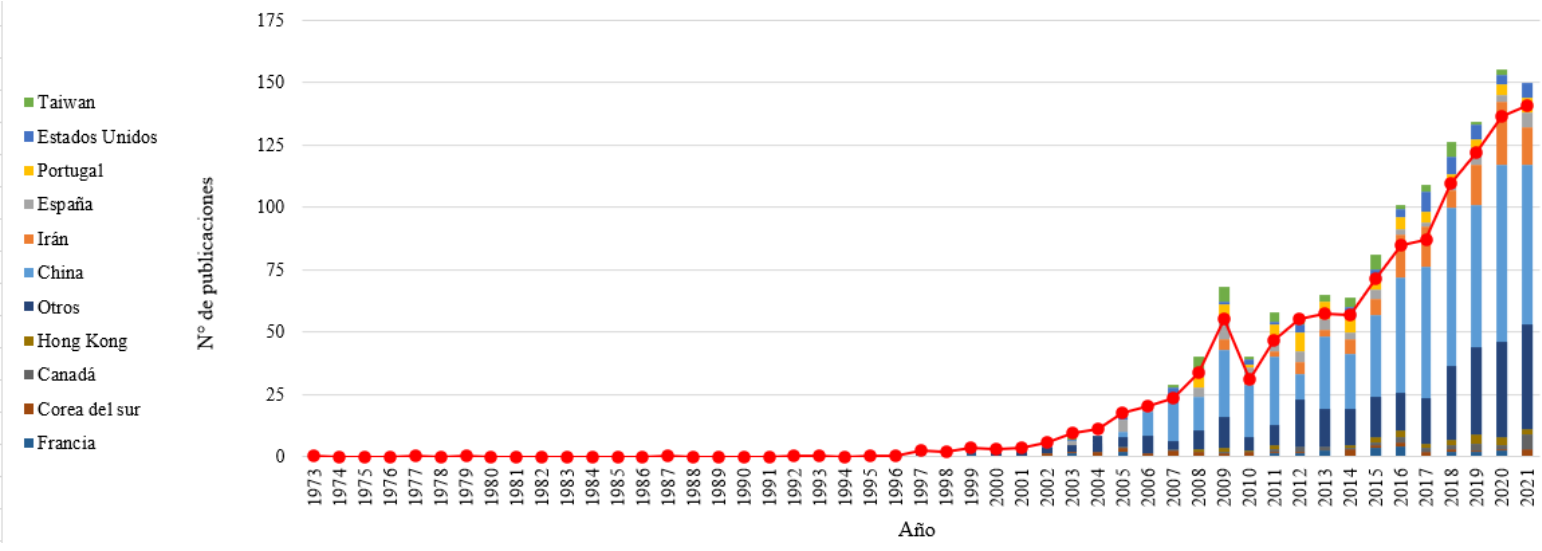

(a)

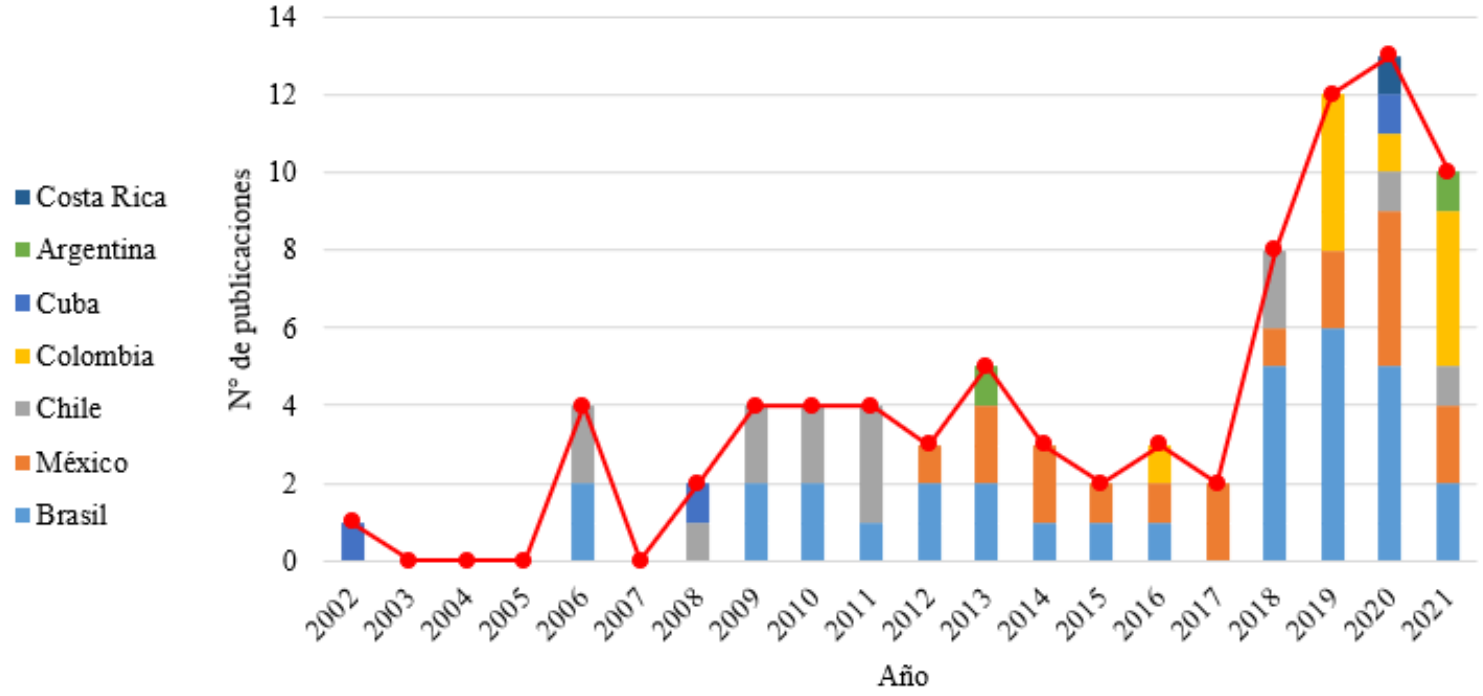

(b)

Figura 6. $N^{\circ}$ de publicaciones por año y por país relacionados con OC (a) en el mundo (b) en América Latina. Fuente: Scopus y SciELO Index

\subsubsection{Principales conceptos: palabras clave $y$ tipos de CECs}

La Figura 7 y 8 muestra la red bibliométrica de palabras clave con un número de co-ocurrencia mínimo de 10 para conceptos clave en OC y tipos de CECs, respectivamente. Para la Figura 7 se identificaron un total de 562 palabras clave con 3 de ellas que superaron las 1000 ocurrencias: "ozone", "catalytic ozonation" y "ozonization". Con más de 500 ocurrencias se identificaron "catalysis", "ozone water treatment" y "ozonation". Otras importantes fueron "degradation", "catalyst activity", "adsorption", "pH" y "hydroxil radical". El tamaño del punto dentro del cluster varía dependiendo del grado de ocurrencia y la intensidad de los vínculos indican el grado de correlación entre los términos (basado en el $\mathrm{n}^{\circ}$ de documentos en los que estos términos aparecen juntos).

Se observa una fuerte correlación entre los términos "ozonization" y "catalytic ozonation" con "wastewater treatment", "hydroxil radicals" y "degradation", lo que sugiere que desde el año 2010 al presente la investigación se ha centrado en mejorar los rendimientos de ozonización a través de la actividad catalítica y la generación de radicales $\bullet \mathrm{OH}$. 


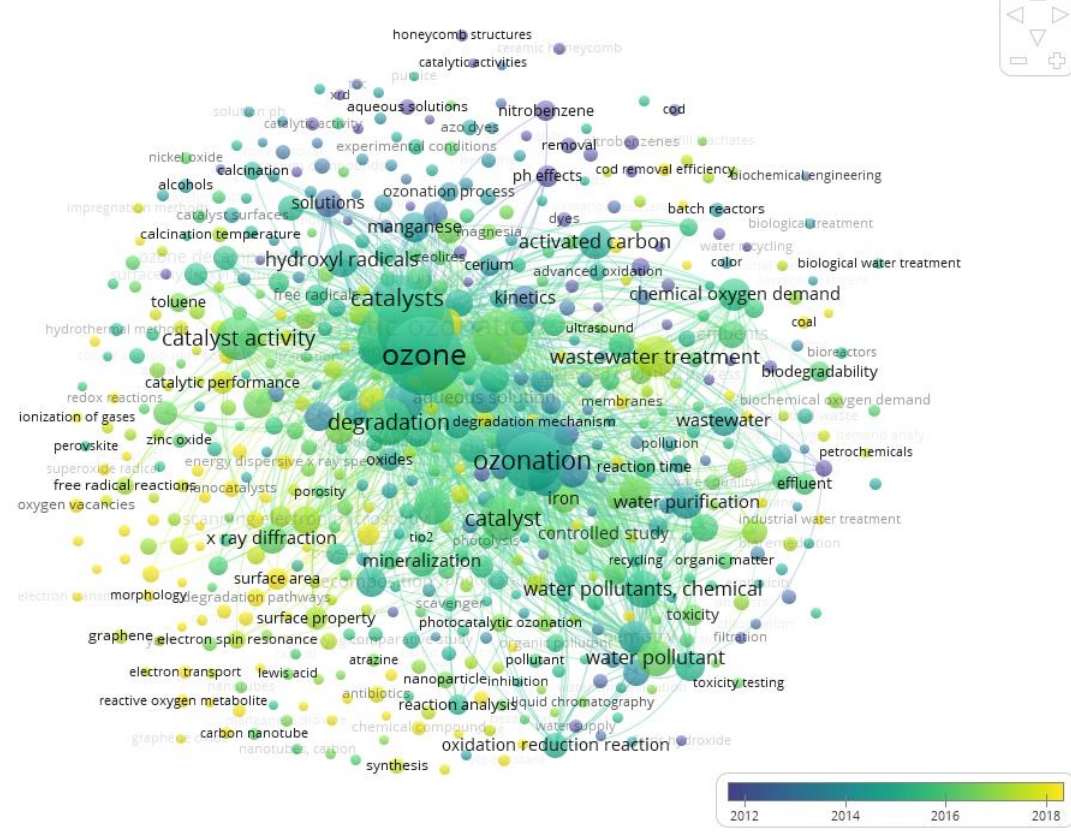

Figura 7. Co-ocurrencia de palabras clave. Los colores asignados se basan en el promedio de publicaciones por año. Fuente: Scopus

Entre los años 2010 y 2016 se identificaron palabras como "catalyst activity", "oxides", " $\mathrm{TiO}_{2}$ ", "Iron", "manganese", "cerium", "activated carbon", "zeolites" y "graphene" lo que sugiere el estudio de óxidos metálicos como catalizadores y su combinación con otros materiales como el CA, las zeolitas y materiales no metálicos, como el grafeno; mientras que desde el 2017 aproximadamente, se han identificado investigaciones relacionadas con el área superficial del catalizador y resonancia electrónica de spin (ESR). Finalmente, la aparición de "photocatalytic ozonation", "membranes" y "ultrasound", aproximadamente desde el año 2015 al presente implica que las investigaciones alrededor de la OC se están combinando con otras tecnologías que permitan mejorar su efectividad.

En cuanto a los tipos de CECs, se construyó la Figura 8 , en donde se reúnen los principales 30 compuestos que aparecen en estudios de OC desde 1973 al 2021. Se observa que el primer contaminante en aparecer en los estudios experimentales fue el ácido acético en el año 1979. El ácido oxálico es el contaminante más investigado, con un total de 125 ocurrencias desde el año 1995, sin embargo, se reporta un descenso en el grado de ocurrencia desde el año 2018. Otros ácidos carboxílicos, como el ácido húmico y ácido clorobenzoico aparecen en las investigaciones. En segundo lugar, se encuentra el fenol (109) y el butanol (92) comenzando desde el año 1987 y 2004, respectivamente. Durante el año 2021, el contaminante con más ocurrencias fue el tolueno (17), seguido del fenol, el ácido húmico y el benceno. Los CECs de procedencia industrial como el bisfenol y los ftalatos se identifican desde el año 2012 y 2006, respectivamente. Otros CECs de tipo farmaceútico son el ibuprofeno, el diclofenaco, la atrazina y el ácido salicílico, los cuales se identifican desde los años 1999, 2008 y 2009 con ocurrencias en aumento, especialmente en el caso de la atrazina y el ibuprofeno para el año 2021. Finalmente, se identifican estudios con pigmentos azoicos, azul de metileno y anilina desde los años 2007 y 2009. 


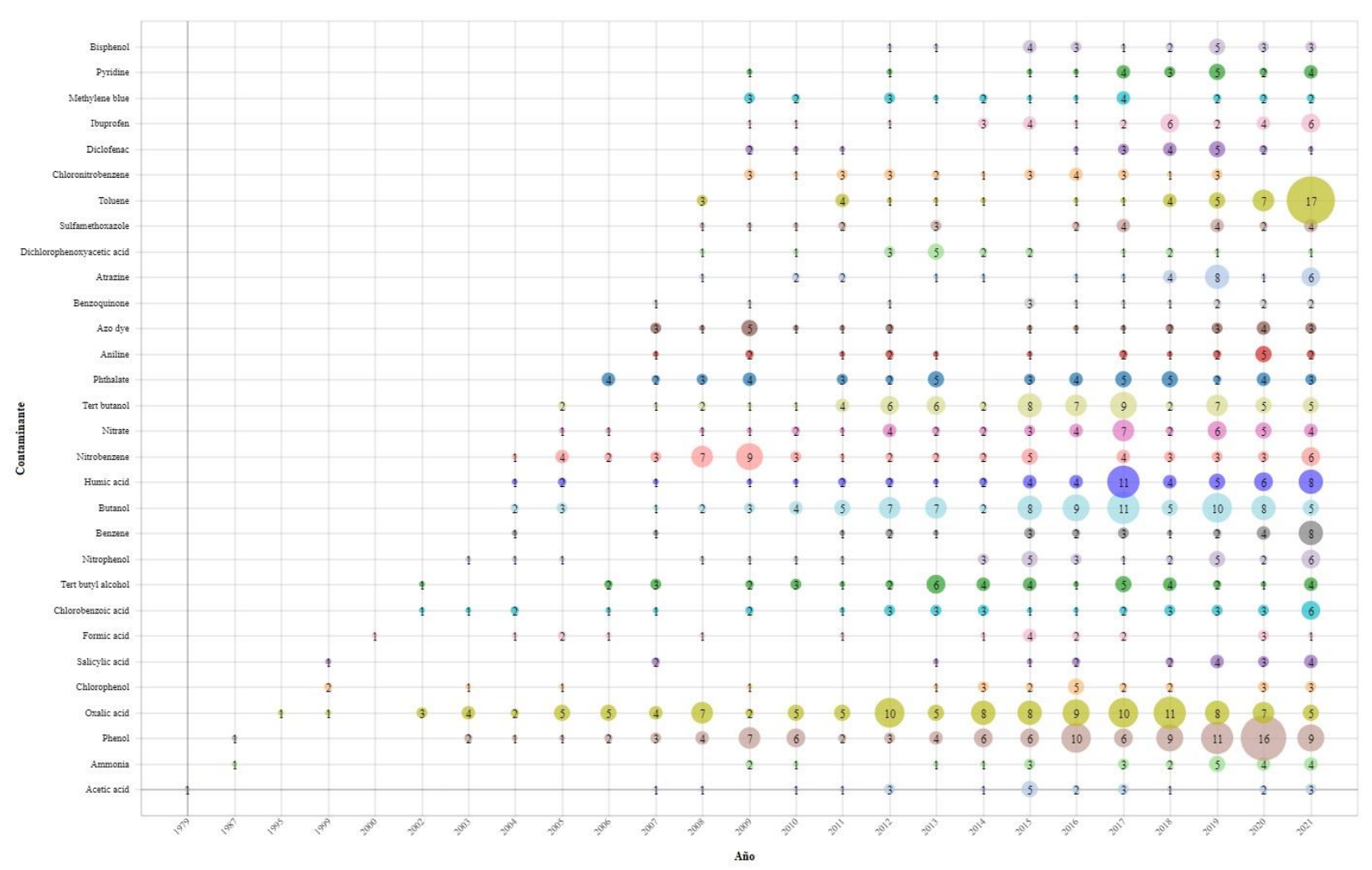

Figura 8. Tipos de contaminante más investigados en OC. Fuente: Scopus

\subsubsection{Principales autores}

Para realizar el análisis de autoría se tomaron en cuenta autores con un mínimo de 5 publicaciones y 20 citaciones. El tamaño del punto indica el autor con más publicaciones, la agrupación en clústeres indica el índice de co-autoría y el tamaño del vínculo la relación entre los autores. En la Figura 9a, el clúster central muestra un gran $\mathrm{n}^{\circ}$ de autores de nacionalidad china que a su vez son los que más publicaciones tienen, entre ellos, Ma J, Zhang $\mathrm{T}$ y Zhao L (entre los años 20102014), y otros como Chen Z, Zhang Y, Zhang J, Wang $J$ y Liu $Y$ desde el año 2015 aproximadamente. Entre ellos, el que más citaciones y publicaciones tiene en el área es $\mathrm{Ma}$ $\mathrm{J}$, con un total de 90 publicaciones y 3061 citaciones, sin embargo, Kasprzyk-Hodern B, aunque no tiene tantas publicaciones, es el segundo en $\mathrm{n}^{\circ}$ de citaciones (2858), seguido por Nawrocki J (2220). Ma J es un autor que de acuerdo con la Tabla 4, se ha centrado en artículos de investigación científica y tecnológica, mientras que Nawrocki J y Kasprzyk-Hodern B, se han centrado en artículos de revisión, de acuerdo con la Tabla 3. A nivel de Hispanoamérica, Beltrán FJ (España) y Pereira MFR (Portugal) son los autores con más publicaciones (35 y 37, respectivamente) y más citaciones (2122 y 1922, respectivamente). En América Latina, los autores más importantes son Poznyak T (México) y Valdés H (Chile), con 15 y 12 publicaciones, 196 y 314 citaciones respectivamente.

Por otro lado, realizando un zoom de autores latinoamericanos, se tomaron en cuenta autores con un mínimo de 2 publicaciones y 5 citaciones por artículo, en vista del bajo volumen de producción científica respecto de la OC. La Figura 9b muestra los autores principales, observándose que los clústeres con puntos más grandes son los de Poznyak T, que tiene el grupo de autores con mayor número de publicaciones (15) desde el año 2016 aproximadamente; y el de Valdés H (12), con publicaciones alrededor de los 


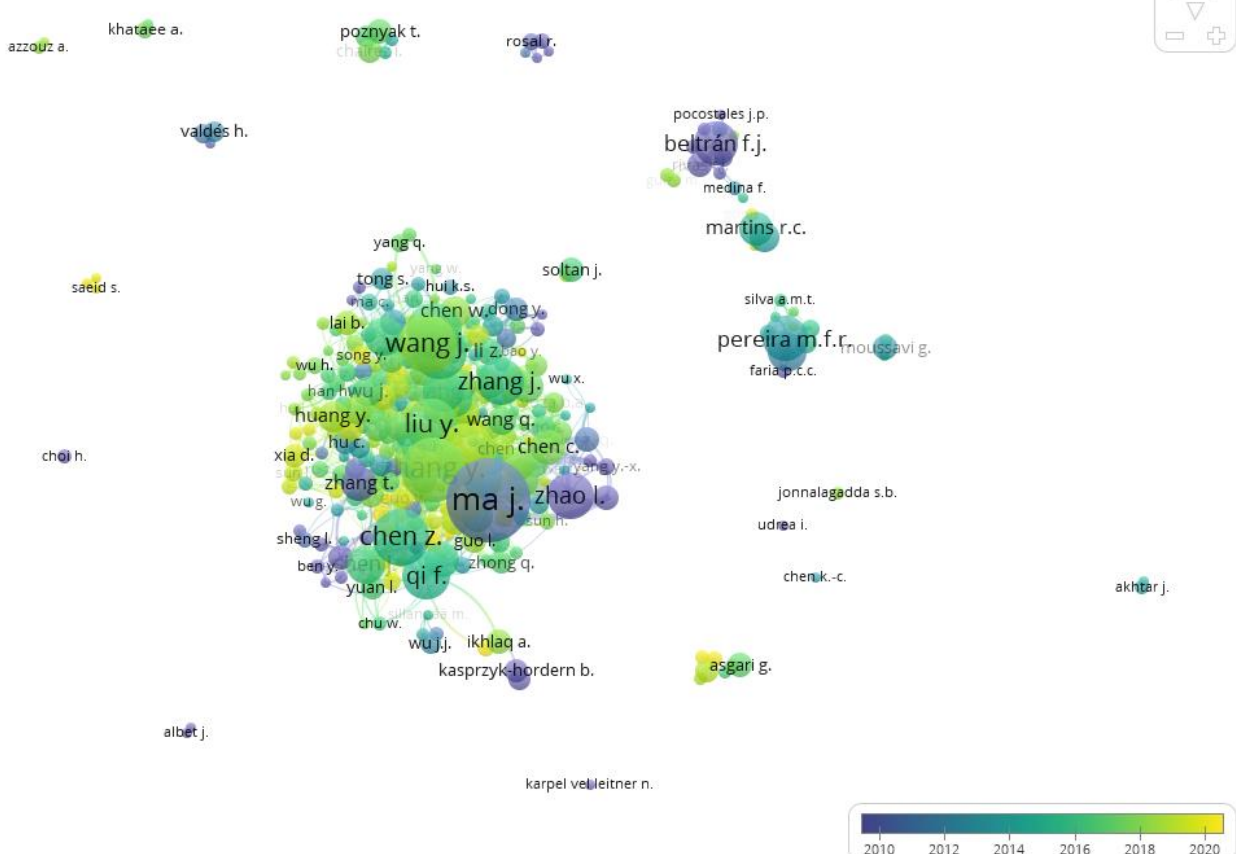

(a)
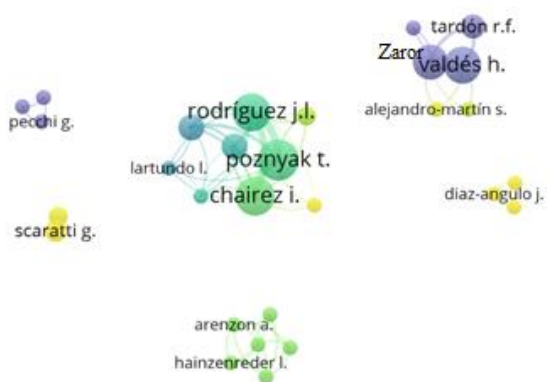

it vosviewer

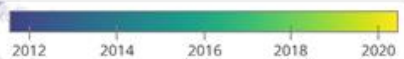

(b)

Figura 9. Análisis de co-autoría y principales autores internacionales (a) Mundo (b) América Latina. Fuente:

Scopus

años 2010 - 2015. Autores más recientes son Scaratti G (4) y Diaz-Angulo J (2), ambos con publicaciones desde el año 2019 aproximadamente. Finalmente, el autor con mayor número de citaciones es Valdés H (314), seguido de Zaror CA (305).

\subsubsection{Principales revistas}

Se analizaron las revistas con más publicaciones sobre OC. La Figura 10a muestra las 3 revistas que más publicaciones tienen a la fecha: Applied Catalysis B: Environmental (96), Chemical
Engineering Journal (82) y Journal of Hazardous Materials (78), manteniendo una media de 3-6 publicaciones por año desde el año 2001 en el caso de Applied Catalysis B: Environmental y Journal of Hazardous Materials, y desde el año 2006 en el caso de Chemical Engineering Journal. Se identificaron otras revistas centradas en el área de ciencias exactas en donde se publican artículos mayormente relacionados con los fundamentos científicos de la OC, son Applied Catalysis B: Environmental, Journal of Hazardous Materials, Chemosphere, Water Science and Technology, 
Huanjing Kexue Environmental Science y Ozone Science and Engineering, siendo esta última en la que se publicaron los primeros artículos de OC (1973). Las revistas que se centran en la aplicación de la tecnología son la Chemical Engineering Journal, Separation and Purification Technology, Journal Of Environmental Chemical Engineering y Desalination and Water treatment, en donde comienzan a aparecer publicaciones que aumentan de manera sostenida en número desde el año 2006 en adelante, lo cual puede significar que la tecnología se está moviendo desde los fundamentos a la aplicación.

La Figura 10b muestra el caso de revistas latinoamericanas, en donde se encontraron solamente 13 publicaciones de OC durante el período 1973-2021. Las revistas con más publicaciones de OC (2) fueron la Brazilian Journal of Chemical Engineering, Ambiente e
Agua, Química Nova (todas de Brasil) y la Journal of the Chilean Chemical Society (Chile).

Para determinar el impacto y la evolución de las revistas, se seleccionaron las 3 con más publicaciones sobre OC de las Figuras 10a y 10b y se analizaron mediante el índice SJR. Los resultados muestran que Applied Catalysis B es la revista de mayor índice SJR (cuartil Q1), seguida de Chemical Engineering Journal (Q1) y Journal of Hazardous Materials (Q1). En América Latina, las revistas con mayor índice SJR son la Journal of the Brazilian Chemical Society (Q3), Brazilian Journal of Chemical Engineering (Q3) y la Journal of the Chilean Chemical Society (Q3). Los resultados de este análisis se muestran en la Figura 11. Finalmente, las Tablas 5 y 6 muestran el índice SJR, el cuartil y el país de las 5 revistas principales a nivel mundial y en América Latina al año 2021, respectivamente.

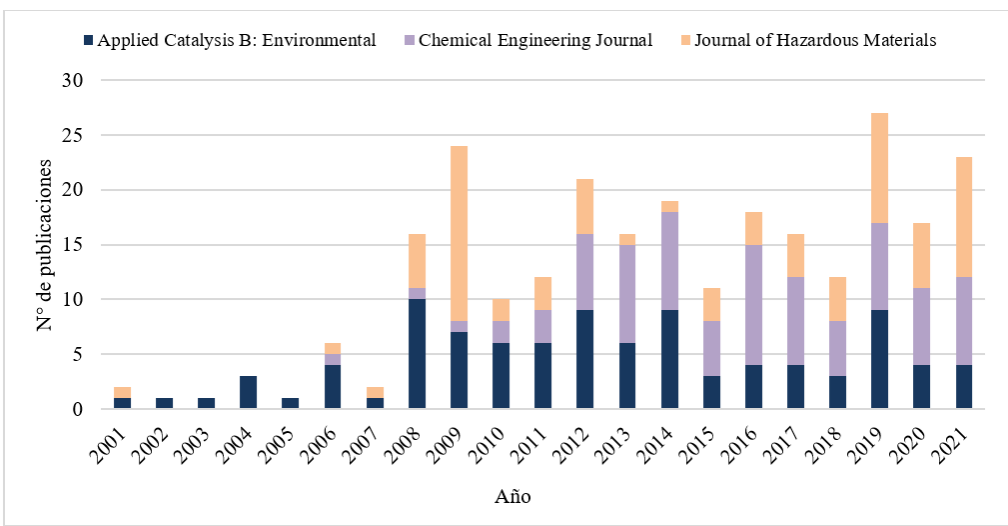

(a)

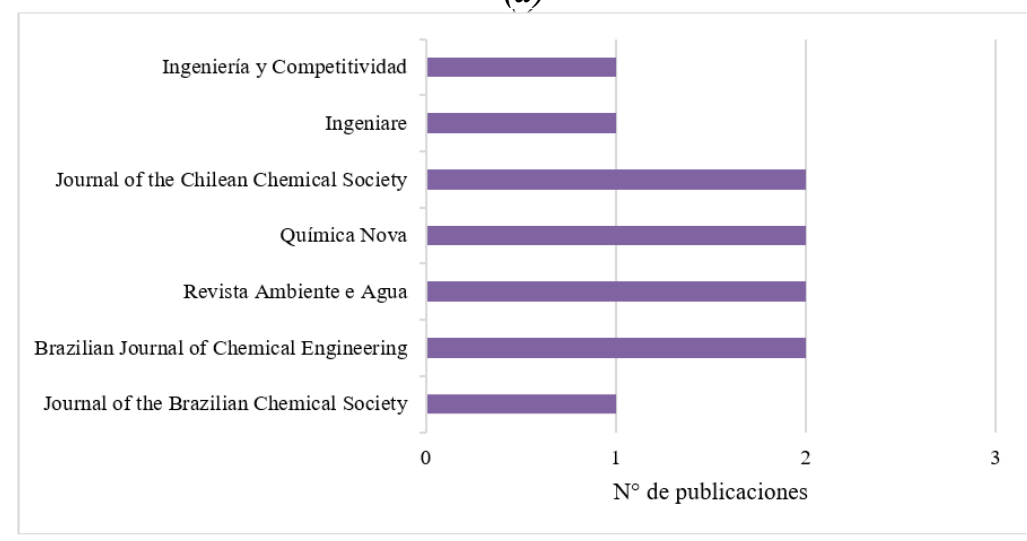

(b)

Figura 10. Principales revistas que publican sobre OC. Fuente: propia con información de Scopus 


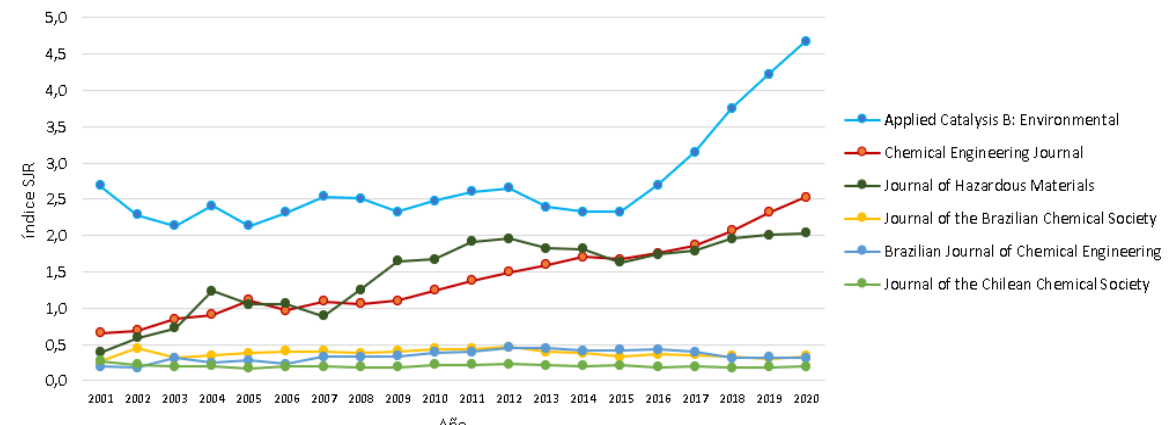

Figura 11. Evolución del índice SJR. Solo se obtuvieron datos desde el año 2001 en adelante. Fuente: SJR, Scopus y Web of Science

Tabla 5. Top 5 revistas científicas que publican sobre OC por índice SJR para el año 2021

\begin{tabular}{cccc}
\hline Nombre de la revista & Índice SJR & Cuartil & País \\
\hline Applied Catalysis B: Environmental & 4.67 & $\mathrm{Q} 1$ & Países bajos \\
Chemical Engineerging Journal & 2.53 & $\mathrm{Q} 1$ & Países bajos \\
Journal of Hazardous Materials & 2.03 & $\mathrm{Q} 1$ & Países bajos \\
Chemosphere & 1.63 & $\mathrm{Q} 1$ & Reino Unido \\
Separation and Purification Technology & 1.28 & $\mathrm{Q} 1$ & Países bajos \\
\hline
\end{tabular}

Fuente: Scimago Journal Rank

Tabla 6. Top 5 revistas científicas latinoamericanas que publican sobre OC por índice SJR para el año 2021

\begin{tabular}{cccc}
\hline Nombre de la revista & Índice SJR & Cuartil & País \\
\hline Journal of the Brazilian Chemical Society & 0.34 & Q3 & Brasil \\
Brazilian Journal of Chemical Engineering & 0.31 & Q3 & Brasil \\
Revista Ambiente e Agua & 0.27 & Q3 & Brasil \\
Química Nova & 0.21 & Q3 & Brasil \\
Journal of the Chilean Chemical Society & 0.20 & Q3 & Chile \\
\hline
\end{tabular}

Fuente: Scimago Journal Rank

Se observa que el cuartil más alto de revistas latinoamericanas que publican sobre OC. De igual manera, el volumen de producción científica sobre esta tecnología está en etapas tempranas en la región, lo cual se observa en el $n^{\circ}$ de artículos publicados. En América Latina la regulación y caracterización de CECs es prácticamente inexistente ${ }^{(84)}$. Este es un motivo importante para explicar el bajo $\mathrm{n}^{\circ}$ de publicaciones en comparación con Europa y Estados Unidos, en donde la regulación de CECs viene estableciéndose y desarrollándose desde antes de la década de los $200{ }^{(85)}$. Los reportes realizados en el año 2015 y 2020 por el Banco Interamericano de Desarrollo y el Banco Mundial concluyen en que, si bien se han hecho muchos esfuerzos alrededor de la problemática de las aguas residuales en América Latina, la región todavía afronta graves problemas respecto al (i) marco legal e institucional, (ii) cobertura de servicios, (iii) tratamiento de aguas residuales, (iv) eficiencia operativa y (v) participación de la sociedad civil y autoridades locales ${ }^{(14,86)}$.

\subsubsection{Principales países}

La Figura 12 muestra el análisis de coautoría por país. Se tuvieron en cuenta publicaciones con un mínimo de 5 citaciones por país. La dimensión de los puntos depende del número de documentos publicados por autores pertenecientes al país relacionado. Los vínculos entre los elementos indican la relación entre los países, que se 
determina en función del número de documentos en coautoría y el grosor del enlace indica la cantidad de vínculos de coautoría entre los países. La variación del color y la línea de años indica el $\mathrm{n}^{\circ}$ de citaciones recibidas por los artículos publicados.

En la Figura 12a, de los 65 países analizados, 35 cumplieron con las condiciones establecidas. El más alto $\mathrm{n}^{\circ}$ de publicaciones lo tiene China (902), seguido por Irán (123), España (73), Portugal (65) y Estados Unidos (59). Por otro lado, el país con mayor cantidad de redes de co-autoría es China
(127 con 18 países, siendo Australia, Estados Unidos y Hong Kong los más importantes), seguido por Estados Unidos (55 con 10 países, siendo China, Canadá y Reino Unido los más relacionados), Hong Kong (37), Australia (29) y España (28). Los colores son indicativos tanto de la antigüedad de la co-autoría como del $\mathrm{n}^{\circ}$ de citas recibidas, ya que los datos están ponderados por el número promedio de citas que recibió el total de artículos de un país. China (19088), España (3204) y Portugal (2685) son los países que produjeron artículos con mayor número medio de citaciones.

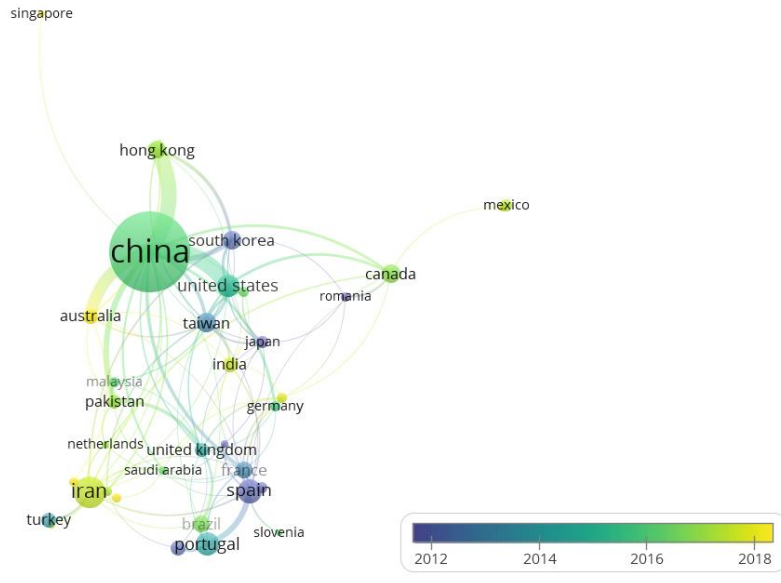

(a)

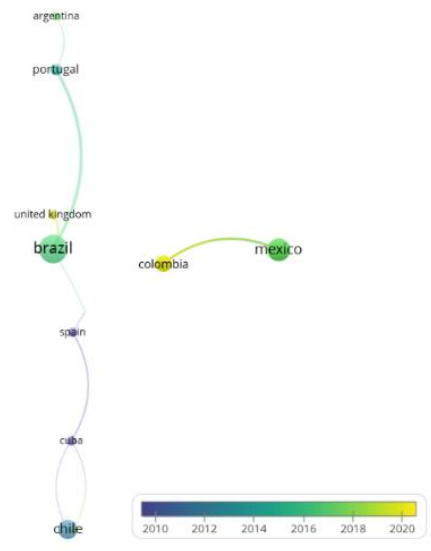

(b)

Figura 12. Análisis de co-autoría por país (a) Mundo (b) América Latina. Fuente: Scopus

Para el caso de América Latina, en la Figura 12b se observa que el mayor $n^{\circ}$ de publicaciones pertenecen a Brasil (31) y México (17). Brasil tiene redes de coautoría en orden de importancia con Portugal, España y Reino Unido (las más recientes), mientras que México tiene relaciones de coautoría principalmente con Colombia. Chile, por su parte, tiene redes de coautoría con Cuba y Argentina con Portugal. El mayor $n^{\circ}$ de citaciones corresponde a Brasil (422), seguido por Chile (328) y México (209), sin embargo, se observa que el promedio de publicaciones chilenas se encuentra alrededor de los años 2010-2013, mientras que Brasil y México tienen publicaciones más recientes (años 2016-2018).
Colombia es un país que desde el año 2019 ha tomado importancia en el panorama de OC, como lo indican también las Figuras $6 \mathrm{~b}$ y $9 \mathrm{~b}$ (con el cluster del autor Díaz-Angulo J).

\subsection{Curva $S$}

Para determinar tendencias y la madurez en publicaciones sobre OC a nivel mundial, se realizó la curva $S$ a través de la Ec. 2. Se seleccionó el modelo logístico porque fue el que se ajustó mejor a los datos y presentó el mejor valor de $\mathrm{R}^{2}$. La Figura 13a muestra la curva $\mathrm{S}$ de OC en el período analizado y se observa que, en términos de artículos científicos, la tecnología se encuentra en la etapa de crecimiento. La línea 
punteada indica el punto de inflexión, en el cual se presenta el cambio de signo de la curva y comienza la etapa de madurez. Se determinó que este punto corresponde a un valor de 1671 publicaciones acumuladas, valor que se alcanzó durante el año 2021, lo cual indica que, según el modelo elegido, la tecnología está ingresando en el período de madurez en términos de publicaciones, sin embargo, todavía requiere mucho más tiempo antes de llegar a la etapa de saturación, lo cual implica un gran potencial de innovación y desarrollo. De igual forma, la curva indica que la OC podría desarrollarse completamente en términos de investigación académica e innovación en los próximos 20 a 30 años, aproximadamente.

La Figura 13b muestra un zoom en la situación de América Latina y se observa que la curva que mejor se ajusta es una de 2 olas. Este tipo curvas son características de cambios en la investigación,

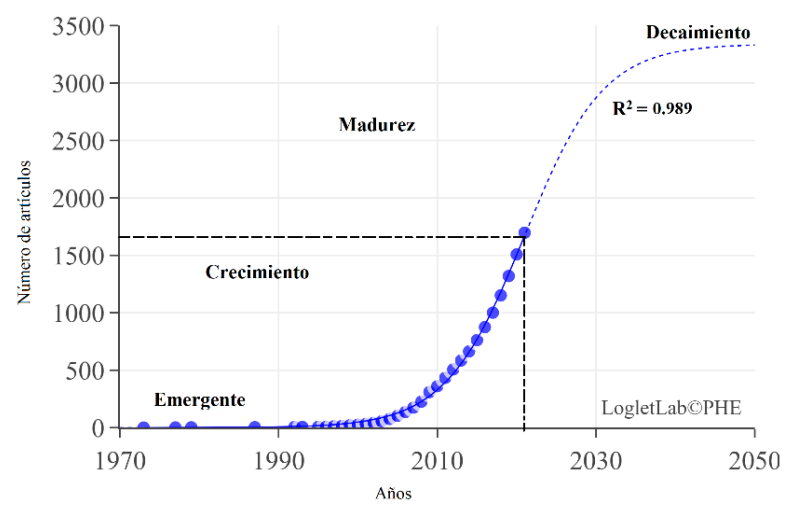

(a) en donde la curva 1 (curva azul) indica que desde el 2002 hasta el 2013 aproximadamente, la investigación pasó su punto de inflexión y entró en declive hasta el 2015, y desde el 2015 (curva verde), se identifica que existe un nuevo enfoque, el cual se encuentra en etapa emergente, que está evolucionando a un ritmo productivo $\mathrm{y}$ prometedor. Esto podría explicarse con los análisis realizados anteriormente, como los vistos en la Figura $6 b, 9 b$ y $12 b$, en donde se identificaron nuevos autores y países que están entrando en el panorama de investigación de OC. Los artículos identificados en países latinoamericanos desde el año 2015 muestran que las investigaciones en OC se están desplazando desde el enfoque de mecanismos y cinéticas de reacción a estudios en donde la OC se combina con otras tecnologías, como la ozonización fotocatalítica, coagulación/floculación, membranas poliméricas y otros catalizadores, especialmente a escala nano ${ }^{(87-89)}$.

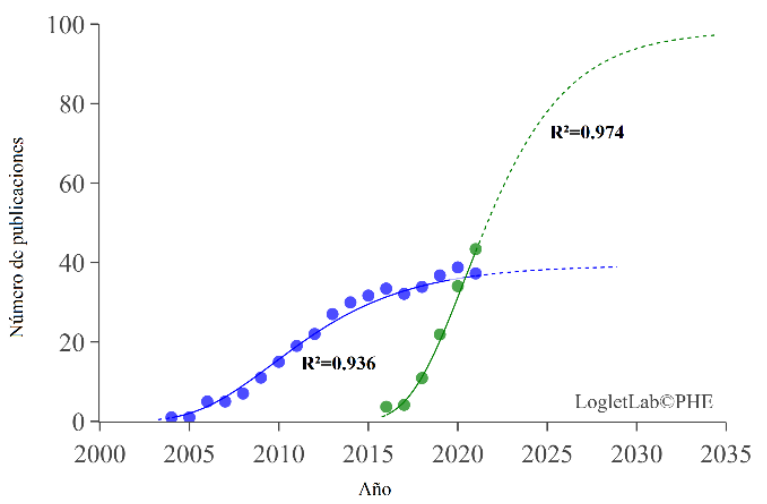

(b)

Figura 13. Curva S de OC a partir del análisis bibliométrico (a) Mundo (b) América Latina. Fuente: LogletLab y Scopus

\section{Conclusiones}

La OC como tecnología en el tratamiento de aguas residuales (domésticas e industriales) ha recibido importante financiación en los últimos años, lo cual se ha visto traducido en un aumento exponencial de la producción bibliográfica, sin embargo, aún quedan muchos retos por afrontar para su implementación industrial.
Se observa que hay muy pocos estudios de OC a escala piloto y en aguas reales, siendo la mayor parte de la investigación aplicada en matrices de agua preparada con sustancias químicas de grado analítico a nivel de laboratorios.

Las desventajas de la OC hasta ahora identificadas radican en la generación de subproductos, el rendimiento en aguas reales y en 
que todavía no se conoce con exactitud el mecanismo de reacción que tiene lugar, sin embargo, para tratar de incrementar los rendimientos y atenuar sus desventajas se ha venido combinando con otros tratamientos como las membranas, tratamientos biológicos, ultrasonido, fenton, radiación UV (ozonización fotocatalítica), entre otras.

Otras investigaciones recientes se han centrado en sintetizar catalizadores a escala nano con nuevos materiales para aumentar el rendimiento del proceso y disminuir los costos operativos. Algunos de los materiales más novedosos son los óxidos de grafeno, espumas metálicas hechas con impresoras 3D, soportes de tipo película polimérica, zeolitas, CA modificado, minerales modificados con metales como las perovskitas y la goetita, entre otros.

El análisis bibliométrico demostró que China es el país más importante, con una contribución del $47 \%$ a la producción bibliográfica mundial de OC, mientras que en América Latina el país más importante es Brasil, con una contribución del 1.5. En lo referente a tipos de contaminantes, se identificó que los más investigados son el ácido oxálico y el fenol, mientras que de los CECs, el ibuprofeno, la atrazina, el ácido salicílico y el bisfenol son algunos de los que más ocurrencias reportaron.

Finalmente, la curva $\mathrm{S}$ de la tecnología mostró a nivel mundial, que la OC se encuentra en la etapa de crecimiento, con gran potencial para la $\mathrm{I}+\mathrm{D}$, mientras que en América Latina está en etapa emergente desde el año 2015.

\section{Referencias}

(1) UN CEPAL. La Agenda 2030 y los Objetivos de Desarrollo Sostenible: una oportunidad para América Latina y el Caribe. Objetivos, metas e indicadores mundiales [Internet]. Comisión Económica para América Latina y El Caribe. 2019. Available from: https://www.cepal.org/es/publicaciones/40155la-agenda-2030-objetivos-desarrollosostenible-oportunidad-america-latina-caribe.
(2) Urdaneta-Carruyo E. Siglo XX. Cien años de infortunio $y$ esplendor. Gac Méd Méx. 2005;141(1):75-84.

(3) ONU. Agua para un mundo sostenible: Datos y cifras. In: Informe de las Naciones Unidas sobre los recursos hídricos en el mundo [Internet]. 2015. p. 1-12. Available from: http://www.unesco.org/new/fileadmin/MULTI MEDIA/HQ/SC/images/WWDR2015Facts_Fi gures_SPA_web.pdf.

(4) Tsiarta N. C.O.VI.D.: Catalytic Ozonation for VIrus Disinfection - Why Not? Nowelties European Joint Doctorade [Internet]. 2020; Available from: https://nowelties.eu/c-o-vi-dcatalytic-ozonation-for-virus-disinfection-whynot/1727/.

(5) Jaramillo MF, Zea DAC, Galvis A. Reutilización de las aguas residuales municipales como estrategia de prevención y control de la contaminación hídrica. Caso de estudio: Cuencas de los ríos Bolo y Frayle (Colombia). Ing y Compet [Internet]. 2020;22(2):1-21.

https://doi.org/10.25100/iyc.v22i2.9412.

(6) Vargas-Pineda OI, Trujillo-González JM, Torres-Mora MA. Huella hídrica: Una herramienta eficaz para el desafío de la sostenibilidad del agua. Ing y Compet [Internet]. 2020;22(1):1-12. https://doi.org/10.25100/iyc.v22i1.8429.

(7) SUEZ. El potencial de la reutilización de las aguas residuales, en una infografía [Internet]. ACTUALIDAD. 2017. Available from: https://www.retema.es/noticia/el-potencial-dela-reutilizacion-de-las-aguas-residuales-enuna-infografia-SfzKX

(8) Porta-Díaz A. Regeneración y reutilización de aguas residuales depuradas [Tesis de maestría]. Universidad Politécnica de Cataluña; 2005. Available from: http://hdl.handle.net/2099.1/5209.

(9) Rosal R, Rodríguez A, Perdigón-Melón JA, Petre A, García-Calvo E, Fernández- AR, et al. Occurrence of emerging pollutants in urban wastewater and their removal through biological treatment followed by ozonation. Water Res [Internet]. 2010;44(2):578-88. https://doi.org/10.1016/j.watres.2009.07.004

(10) Rice RG, Robson M, Miller W, Hill AG. Uses of ozone in drinking water treatment. J AWWA [Internet]. 1981;73(1):44-57. https://doi.org/10.1002/j.15518833.1981.tb04637.x

(11) Rojas JAA, Giraldo LFG, Ruíz ÁA. Mineralización del colorante Tartrazina 
mediante un proceso de oxidación avanzada. Rev LASALLISTA Investig. 2009;6(2):46-53.

(12) Dewil R, Mantzavinos D, Poulios I, Rodrigo MA. New perspectives for Advanced Oxidation Processes. J Environ Manage [Internet]. 2017;195(2):93-9.

https://doi.org/10.1016/j.jenvman.2017.04.010

(13) Wang J, Chen H. Catalytic ozonation for water and wastewater treatment: Recent advances and perspective. Sci Total Environ [Internet]. 2020;704:135249.

https://doi.org/10.1016/j.scitotenv.2019.13524 9

(14) Jeirani Z. Jeirani Z. Catalytic ozonation of emerging pollutants in water in the presence of MCM-41 and Fe-MCM-41 [Internet]. University of Saskatchewan; 2018. Available from: http://hdl.handle.net/10388/9242

(15) Rodriguez DJ, Serrano HA, Delgado A, Nolasco D, Saltiel G. De residuo a recurso [Internet]. Washington, DC; 2020. Available from:

https://www.bancomundial.org/es/topic/water/ publication/wastewater-initiative

(16) Donthu N, Kumar S, Mukherjee D, Pandey N, Lim WM. How to conduct a bibliometric analysis: An overview and guidelines. J Bus Res [Internet]. 2021;133:285-96. https://doi.org/10.1016/j.jbusres.2021.04.070

(17) Scite_ [Internet]. Brooklyn, NY: National Science Foundation, National Institutes of Health; Available from: https://scite.ai/search

(18) Leiden University. VOSviewer [Internet]. Leiden, Netherlands: Leiden University; 2021. Available from: https://www.vosviewer.com/download

(19) Scimago Lab. WHAT IS SCIMAGOJR FOR? [Internet]. scimagojr.com. 2007 [cited 2021 Sep 4]. Available from: https://www.scimagojr.com/.

(20) Taylor M, Taylor A. The technology life cycle: Conceptualization and managerial implications. Int J Prod Econ. 2012;140(1):541-553. https://doi.org/10.1016/j.ijpe.2012.07.006

(21) The Rockefeller University. Loglet Analysis [Internet]. phe.rockefeller.edu. 2010 [cited 2021 Sep 4]. Available from: https://logletlab.com/?page=index\&preload=li brary.get.1.

(22) Beltrán FJ, Rey A, Gimeno O. The Role of Catalytic Ozonation Processes on the Elimination of DBPs and Their Precursors in Drinking Water Treatment. Catalysts [Internet]. 2021;11:521. https://doi.org/10.3390/catal11040521
(23) Geissen V, Mol H, Klumpp E, Umlauf G, Nadal $\mathrm{M}$, Ploeg $\mathrm{M}$ van der. Emerging pollutants in the environment: A challenge for water resource management. Int Soil Water Conserv Res [Internet]. 2015;3(1):57-65. https://doi.org/10.1016/j.iswcr.2015.03.002

(24) Hoigné J. Chemistry of aqueous ozone and transformation of pollutants by ozonation and advanced oxidation processes. In: Hrubec J, editor. Quality and treatment of drinking water II - The Handbook of Environmental Chemistry Vol 5 Part C [Internet]. Heidelberg: SpringerVerlag; $1998 . \quad$ p. 83-141. https://doi.org/10.1007/978-3-540-68089-5_5

(25) Sáenz B. Caracterización de arena impregnada con $\mathrm{TiO} 2$ como catalizador heterogéneo en el proceso de ozonización de aguas contaminadas con naproxeno, ketoprofeno y cimetidina [Internet]. Universidad de Costa Rica; 2019. Available from: http://repositorio.sibdi.ucr.ac.cr:8080/jspui/han dle/123456789/9145

(26) Kasprzyk-Hordern B, Ziółek M, Nawrocki J. Catalytic ozonation and methods of enhancing molecular ozone reactions in water treatment. Appl Catal B Environ [Internet]. 2003;46(4):639-69. https://doi.org/10.1016/S0926-3373(03)003266

(27) Rodríguez JL, Fuentes I, Aguilar CM, Valenzuela MA, Poznyak T, Chairez I. Catalytic Ozonation as a Promising Technology for Application in Water Treatment: Advantages and Constraints. In: Derco J, Koman M, editors. Ozone in Nature and Practice [Internet]. IntechOpen; 2018. p. 883912. Available from: https://doi.org/10.5772/intechopen.76228

(28) Beltrán FJ, Rivas FJ, Montero-de-Espinosa R. Ozone-Enhanced Oxidation of Oxalic Acid in Water with Cobalt Catalysts. 1. Homogeneous Catalytic Ozonation. Ind Eng Chem Res [Internet]. 2003;42(14):3210-3217. https://doi.org/10.1021/ie0209982

(29) Glaze WH, Kang J-W, Chapin DH. The Chemistry of Water Treatment Processes Involving Ozone, Hydrogen Peroxide and Ultraviolet Radiation. Ozone Sci Eng [Internet]. 1987;9(4):335-52. https://doi.org/10.1080/01919518708552148

(30) Legrini O, Oliveros E, Braun AM. Photochemical processes for water treatment. Chem Rev [Internet]. 1993;93(2):671-698. https://doi.org/10.1021/cr00018a003

(31) Hill GR. The Kinetics of the Oxidation of Cobaltous Ion by Ozone. J Am Chem Soc 
[Internet].

1949;71(7):2434-2435. https://doi.org/10.1021/ja01175a056

Hewes C., Davison R. Renovation of wastewater by ozonation. Water. 1973;71-80.

Wang J, Xu LJ. Advanced Oxidation Processes for Wastewater Treatment: Formation of Hydroxyl Radical and Application. Crit Rev Environ Sci Technol [Internet]. 2012;42(3):251-325.

https://doi.org/10.1080/10643389.2010.507698

(34) Khuntia S, Kumar-Sinha M, Singh P. Theoretical and experimental investigation of the mechanism of the catalytic ozonation process by using a manganese-based catalyst. Environ Technol [Internet]. 2021;42(4):632-9. https://doi.org/10.1080/09593330.2019.164080 0

(35) Nawrocki J. Catalytic ozonation in water controversies and questions. Discussion paper. Appl Catal B Environ [Internet]. 2013;142143:465-71.

https://doi.org/10.1016/j.apcatb.2013.05.061

(36) Ziylan A, Ince NH. Catalytic ozonation of ibuprofen with ultrasound and Fe-based catalysts. Catal Today [Internet]. 2015;240(Part A):2-8.

https://doi.org/10.1016/j.cattod.2014.03.002

Yildirim AO, Gul S, Eren O, Kusvuran E. A Comparative Study of Ozonation, Homogeneous Catalytic Ozonation, and Photocatalytic Ozonation for C.I. Reactive Red 194 Azo Dye Degradation. Clean - Soil, Air, Wate [Internet]. 2011;39(8):795-805. https://doi.org/10.1002/clen.201000192

(38) Nawrocki J, Kasprzyk-Hordern B. The efficiency and mechanisms of catalytic ozonation. Appl Catal B Environ [Internet]. 2010;99(1-2):27-42.

https://doi.org/10.1016/j.apcatb.2010.06.033

(39) Messias RA, Filho HJI, Ferreira GA, Silva OA da, Siqueira AF. Catalytic Ozonation Using Fe2+in the Treatment of Dairy Effluent in a Semi-Batch Process with Recycle. J Braz Chem Soc [Internet]. 2015;26(7):1-11. http://dx.doi.org/10.5935/0103-5053.20150120

(40) Wu C-H, Kuo C-Y, Chang C-L. Homogeneous catalytic ozonation of C.I. Reactive Red 2 by metallic ions in a bubble column reactor. J Hazard Mater. 2008;154(1-3):748-55. https://doi.org/10.1016/j.jhazmat.2007.10.087

(41) Zhang Y, Zhao P, Li J, Hou D, Wang J. A hybrid process combining homogeneous catalytic ozonation and membrane distillation for wastewater treatment. Chemosphere2 [Internet]. 2016;160:134-40. https://doi.org/10.1016/j.chemosphere.2016.06. 070

(42) Guo Y, Wang H, Wang B, Deng S, Huang J. Prediction of micropollutant abatement during homogeneous catalytic ozonation by a chemical kinetic model. Water Res [Internet]. 2018;142:383-95.

https://doi.org/10.1016/j.watres.2018.06.019

(43) Malvestiti JA, Cruz-Alcalde A, López-Vinent N, Dantas RF, Sans C. Catalytic ozonation by metal ions for municipal wastewater disinfection and simulataneous micropollutants removal. Appl Catal B Environ [Internet]. 2019;259:118104. https://doi.org/10.1016/j.apcatb.2019.118104

(44) Balcıoğlu IA, Moral ÇK. Homogeneous and Heterogeneous Catalytic Ozonation of Pulp Bleaching Effluent. J Adv Oxid Technol [Internet]. 2016;11(3):543-50. https://doi.org/10.1515/jaots-2008-0314

(45) Crousier C, Pic J-S, Albet J, Baig S, Roustan M. Urban Wastewater Treatment by Catalytic Ozonation. Ozone Sci Eng [Internet]. 2016;38(1):3-13. https://doi.org/10.1080/01919512.2015.111311 9

(46) Yu G, Wang Y, Cao H, Zhao H, Xie Y. Reactive Oxygen Species and Catalytic Active Sites in Heterogeneous Catalytic Ozonation for Water Purification. Environ Sci Technol [Internet]. 2020;54:5931-46.

https://dx.doi.org/10.1021/acs.est.0c00575?ref $=\mathrm{pdf}$

(47) Wang B, Zhang H, Wang F, Xiong X, Tian K. Application of Heterogeneous Catalytic Ozonation for Refractory Organics in Wastewater. Catalysts [Internet]. 2019;9(3):241. https://doi.org/10.3390/catal9030241

(48) Beltrán FJ, Rivas J, Álvarez P, Montero-deEspinosa. Kinetics of Heterogeneous Catalytic Ozone Decomposition in Water on an Activated Carbon. Ozone Sci Eng J Int Ozone Assoc [Internet]. 2007;24(4):227-37. http://dx.doi.org/10.1080/01919510208901614

(49) Huang Y, Cui C, Zhang D, Li L, Pan D. Heterogeneous catalytic ozonation of dibutyl phthalate in aqueous solution in the presence of iron-loaded activated carbon. Chemosphere [Internet]. 2015;119:295-301. http://dx.doi.org/10.1016/j.chemosphere.2014. 06.060

(50) Nemati-Sani O, Navaei AA, Yazdani M, Taghavi M. Catalytic ozonation of ciprofloxacin using $\gamma$-Al2O 3 nanoparticles in synthetic and real wastewaters. J Water Process 
Eng [Internet]. 2019;32:100894. https://doi.org/10.1016/j.jwpe.2019.100894

(51) Li Y, Wu L, Wang Y, Ke P, Xu J, Guan B. $\gamma$ $\mathrm{A} 12 \mathrm{O} 3$ doped with cerium to enhance electron transfer in catalytic ozonation of phenol. J Water Process Eng [Internet]. 2020;36:101313. https://doi.org/10.1016/j.jwpe.2020.101313

(52) Hu E, Shang S, Tao X, Jiang S, Chiu K. Regeneration and reuse of highly polluting textile dyeing effluents through catalytic ozonation with carbon aerogel catalysts. J Clean Prod [Internet]. 2016;137:1055-65. https://doi.org/10.1016/j.jclepro.2016.07.194

(53) Kolosov P, Peyot M-L, Yargeau V. Novel materials for catalytic ozonation of wastewater for disinfection and removal of micropollutants. Sci Total Environ [Internet]. 2018;644:120718.

https://doi.org/10.1016/j.scitotenv.2018.07.022

(54) Polonite Nordic AB. Reactive filter media Polonite $\AA$ [Internet]. What is Polonite $\AA$ ? 2020. Available from: https://polonite.se/aboutpolonite/

(55) Zhang T, Li C, Ma J, Tian H, Qiang Z. Surface hydroxyl groups of synthetic $\alpha-\mathrm{FeOOH}$ in promoting radical dot $\} \mathrm{OH}$ generation from aqueous ozone: Property and activity relationship. Appl Catal B Environ [Internet]. 2008;82(1-2):131-7.

https://doi.org/10.1016/j.apcatb.2008.01.008

(56) Ernst M, Lurot F, JC S. Catalytic ozonation of refractory organic model compounds in aqueous solution by aluminum oxide. Appl Catal B Environ [Internet]. 2004;47(1):15-25. Available from: https://doi.org/10.1016/S09263373(03)00290-X

(57) Beltrán F, Rivas F, Montero-de-Espinosa R. Catalytic ozonation of oxalic acid in an aqueous $\mathrm{TiO} 2$ slurry reactor. Appl Catal B Environ [Internet]. 2002;39(3):221-31. https://doi.org/10.1016/S0926-3373(02)001029

(58) Chen Y-H, Hsieh D-C, Shang N-C. Efficient mineralization of dimethyl phthalate by catalytic ozonation using $\mathrm{TiO} 2 / \mathrm{Al} 2 \mathrm{O} 3$ catalyst. J Hazard Mater [Internet]. 2011;192(3):101725.

https://doi.org/10.1016/j.jhazmat.2011.06.005

(59) Wang Y, Chen L, Chen C, Xi J, Cao H. Occurrence of both hydroxyl radical and surface oxidation pathways in N-doped layered nanocarbons for aqueous catalytic ozonation. Appl Catal B Environ [Internet]. 2019;254:28391.

https://doi.org/10.1016/j.apcatb.2019.05.008
(60) Chen C, Yan X, Yoza BA, Zhou T, Li Y, Zhan $\mathrm{Y}$, et al. Efficiencies and mechanisms of ZSM5 zeolites loaded with cerium, iron, or manganese oxides for catalytic ozonation of nitrobenzene in water. Sci Total Environ [Internet]. 2018;612:1424-32. https://doi.org/10.1016/j.scitotenv.2017.09.019

(61) AlGburi HR, Aziz HA, Zwain HM, Noor AFM. Treatment of Landfill Leachate by Heterogeneous Catalytic Ozonation with Granular Faujasite Zeolite. Environ Eng Sci [Internet]. 2020; ahead of p. https://doi.org/10.1089/ees.2020.0233

(62) Liu Z, Ma J, Cui Y, Zhang B. Effect of ozonation pretreatment on the surface properties and catalytic activity of multi-walled carbon nanotube. Appl Catal B Environ [Internet]. 2009;92(3-4):301-6. https://doi.org/10.1016/j.apcatb.2009.08.007

(63) Afzal S, Quan X, Zhang J. High surface area mesoporous nanocast LaMO3 $(\mathrm{M}=\mathrm{Mn}, \mathrm{Fe})$ perovskites for efficient catalytic ozonation and an insight into probable catalytic mechanism. Appl Catal B Environ [Internet]. 2017;206:692703.

https://doi.org/10.1016/j.apcatb.2017.01.072

(64) Wang Y, Xie Y, Sun H, Xiao J, Cao H. Efficient Catalytic Ozonation over Reduced Graphene Oxide for p-Hydroxylbenzoic Acid (PHBA) Destruction: Active Site and Mechanism. ACS Appl Mater Interfaces [Internet]. 2016;8(15):9710-20. https://doi.org/10.1021/acsami.6b01175

(65) Zhang T, Ma J. Catalytic ozonation of trace nitrobenzene in water with synthetic goethite. J Mol Catal A Chem [Internet]. 2008;279(1):829.

https://doi.org/10.1016/j.molcata.2007.09.030

(66) Wang Y, Xie Y, Sun H, Xiao J, Cao H, Wang S. 2D/2D nano-hybrids of $\gamma-\mathrm{MnO} 2$ on reduced graphene oxide for catalytic ozonation and coupling peroxymonosulfate activation. J Hazard Mater [Internet]. 2016;301:56-64. https://doi.org/10.1016/j.jhazmat.2015.08.031

(67) Deng F, Qiu S, Chen C, Ding X, Ma F. Heterogeneous Catalytic Ozonation of Refinery Wastewater over Alumina-Supported Mn and $\mathrm{Cu}$ Oxides Catalyst. Ozone Sci Eng [Internet]. 2015;37(6):546-55. https://doi.org/10.1080/01919512.2015.106517 3

(68) Zhao L, Sun Z, Ma J. Novel relationship between hydroxyl radical initiation and surface group of ceramic honeycomb supported metals for the catalytic ozonation of nitrobenzene in aqueous solution. Environ Sci Technol 
[Internet]. 2009;43(11):4157-63. https://doi.org/10.1021/es900084w

(69) Xing S, Lu X, Liu J, Zhu L, Ma Z, Wu Y. Catalytic ozonation of sulfosalicylic acid over manganese oxide supported on mesoporous ceria. Chemosphere [Internet]. 2016;144:7-12. Available

from: https://doi.org/10.1016/j.chemosphere.2015.08. 044

(70) Martins R, Quinta-Ferreira R. Catalytic ozonation of phenolic acids over a Mn-Ce-O catalyst. Appl Catal B Environ [Internet]. 2009;90(1-2):268-77.

https://doi.org/10.1016/j.apcatb.2009.03.023

(71) Liotta LF, Gruttadauria M, Di Carlo G, Perrini G, Librando V. Heterogeneous catalytic degradation of phenolic substrates: Catalysts activity. J Hazard Mater [Internet]. 2009;162(23):588-606. https://doi.org/10.1016/j.jhazmat.2008.05.115

(72) Wang J, Bai Z. Fe-based catalysts for heterogeneous catalytic ozonation of emerging contaminants in water and wastewater. Chem Eng J [Internet]. 2016;312:79-98. https://doi.org/10.1016/j.cej.2016.11.118

(73) Legube B, Karpel Vel Leitner N. Catalytic ozonation: A promising advanced oxidation technology for water treatment. Catal Today [Internet]. 1999;53(1):61-72. https://doi.org/10.1016/S0920-5861(99)001030

(74) Faria P, Órfao J, Pereira M. Activated carbon catalytic ozonation of oxamic and oxalic acids. Appl Catal B Environ [Internet]. 2008;79(3):237-43.

https://doi.org/10.1016/j.apcatb.2007.10.021

(75) Einaga H, Futamura S. Catalytic oxidation of benzene with ozone over alumina-supported manganese oxides. J Catal [Internet]. 2004;227(2):304-12.

https://doi.org/10.1016/j.jcat.2004.07.029

(76) Banco Mundial. Un 70\% de las aguas residuales de Latinoamérica vuelven a los ríos sin ser tratadas. Noticias [Internet]. 2013 Jan 2; Available from: https://www.bancomundial.org/es/news/feature /2014/01/02/rios-de-latinoamericacontaminados

(77) Mateo-Sagasta J, editor. Reutilizacion de aguas para agricultura en America Latina y el Caribe: estado, principios y necesidades. [Internet]. Santiago, Chile: FAO; 2013. Available from: https://hdl.handle.net/10568/91293

(78) Reichert G, Hilgert S, Fuchs S, Azevedo JCR. Emerging contaminants and antibiotic resistance in the different environmental matrices of Latin America. Environ Pollut [Internet]. 2019;255(1):113140. https://doi.org/10.1016/j.envpol.2019.113140

(79) Castro J, Paz S, Mena N, Urresta J, MachucaMartinez F. Evaluation of heterogeneous catalytic ozonation process for diclofenac degradation in solutions synthetically prepared. Env Sci Pollut Res [Internet]. 2019;26:44884497. https://doi.org/10.1007/s11356-0182582-1

(80) MINAMBIENTE. Resolución No. 631 de 2015 [Internet]. Colombia; 2015 p. 1-93. Available from:

http://www.emserchia.gov.co/PDF/Resolucion 631.pdf.

(81) Gil MJ, Soto AM, Usma JI, Gutiérrez OD. Contaminantes emergentes en aguas, efectos y posibles tratamientos. Prod + Limpia. 2012;7(2):52-73.

(82) Oliveira JS de, Salla J da S, Kuhn RC, Jahn SL, Foletto EL. Catalytic Ozonation of Melanoidin in Aqueous Solution over CoFe2O4 Catalyst. Mat Res [Internet]. 2019;22(1):e20180405. https://doi.org/10.1590/1980-5373-MR-20180405

(83) Peixoto ALC, Silva MB, Filho HJI. Leachate treatment process at a municipal stabilized landfill by catalytic ozonation: an exploratory study from Taguchi orthogonal array. Braz J Chem Eng [Internet]. 2009;26(3):481-92. https://doi.org/10.1590/S010466322009000300004

(84) Zubillaga A de la S. Tendencias legislativas sobre contaminantes emergentes en Europa. In: JORNADA ESAMUR [Internet]. Consorcio de Aguas de Bilbao Bizkaia; 2016. Available from: https://www.esamur.com/public/file/4JornadaE SAMUR20161107AdlSotarev2.pdf

(85) Emilio Lentini. El futuro de los servicios de agua y saneamiento en América Latina [Internet]. Banco Interamericano de Desarrollo; 2015. Available from: https://publications.iadb.org/publications/spani sh/document/El-futuro-de-los-servicios-deagua-y-saneamiento-en-América-LatinaDesafíos-de-los-operadores-de-áreas-urbanasde-más-de-300000-habitantes.pdf

(86) Fuentes I, Rodríguez J, Poznyak T, Chairez I. Photocatalytic ozonation of terephthalic acid: a by-product-oriented decomposition study. Environ Sci Pollut Res [Internet]. 2014;21:12241-12248. https://doiorg.bd.univalle.edu.co/10.1007/s11356-0143176-1

(87) Rodríguez J, Valenzuela $\mathrm{M}$, Tiznado $\mathrm{H}$, Poznyak T, Flores E. Synthesis of nickel oxide 
nanoparticles supported on $\mathrm{SiO} 2$ by sensitized liquid phase photodeposition for applications in catalytic ozonation. J Mol Catal A Chem [Internet]. 2014;392:39-49. https://doi.org/10.1016/j.molcata.2014.04.028

(88) Lara-Ramos J, Diaz-Angulo J, MachucaMartínez F. Use of modified flotation cell as ozonation reactor to minimize mass transfer limitations. Chem Eng J [Internet]. 2021;405:126978.

https://doi.org/10.1016/j.cej.2020.126978

(89) Bessegato G, Cardoso J, da Silva B, Zanoni MV. Combination of photoelectrocatalysis and ozonation: A novel and powerful approach applied in Acid Yellow 1 mineralization. Appl Catal B Environ [Internet]. 2016;180:161-8. https://doi.org/10.1016/j.apcatb.2015.06.013 